\title{
Diagnostik und Therapie der Immunthrombozytopenie
}

\section{Empfehlungen einer gemeinsamen Expertengruppe der DGHO, DGTI und GTH}

\author{
Axel Matzdorff ${ }^{a} \quad$ Aristoteles Giagounidis $^{b} \quad$ Andreas Greinacher $^{c}$ Erhard Hiller $^{d}$ \\ Volker Kiefel $^{\mathrm{e}}$ Hannes Müller-Beißenhirtz ${ }^{f} \quad$ Helmut Ostermann ${ }^{g}$ Mathias Rummel ${ }^{\mathrm{h}}$ \\ Ulrich J. Sachsi Abdulgabar Salama ${ }^{k}$ \\ a Klinik f. Hämatologie/Onkologie, Caritasklinik St. Theresia, Saarbrücken,

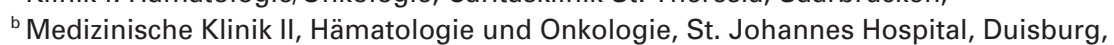 \\ ${ }^{c}$ Institut für Immunologie und Transfusionsmedizin, Ernst-Moritz-Arndt-Universität Greifswald, \\ d Hämatologisch-onkologische Gemeinschaftspraxis, München, \\ ${ }^{e}$ Abteilung für Transfusionsmedizin, Universität Rostock, \\ ${ }^{f}$ Klinik für Hämatologie, Universitätsklinikum Essen, \\ ${ }^{g}$ Medizinische Klinik und Poliklinik III, Klinikum der Universität München-Großhadern, \\ ${ }^{\mathrm{h}}$ Medizinische Klinik IV am Zentrum für Innere Medizin, \\ ' Zentrum für Transfusionsmedizin und Hämotherapie, Institut für Klinische Immunologie und Transfusionsmedizin, Universitätsklinikum \\ Gießen und Marburg, Standort Gießen, \\ ${ }^{k}$ Institut für Transfusionsmedizin, Charité Universitätsklinikum, Campus Virchow Klinikum, Berlin, Deutschland
}

Durch die Einführung der neuen Wirkstoffgruppe der Thrombopoietinrezeptor-Agonisten (TRAs) mussten die bisherigen Leitlinien aus den Jahren 1996, 2003 und 2008 [1-3] überarbeitet werden. Die folgenden Therapieempfehlungen sind das Ergebnis eines Expertentreffens. Sie berücksichtigen alle wesentlichen Studienergebnisse der letzten Jahre inklusive des neuen internationalen Konsensusreports [4], aber auch die besonderen arzneimittelrechtlichen Gegebenheiten in der Bundesrepublik.

\section{Einführung, Definition und Terminologie}

Eine Thrombozytopenie kann Folge einer Störung der Thrombozytenneubildung, eines beschleunigten Abbaus, eines starken Verlusts oder einer Verteilungsstörung sein (Tab. 1). Bei der Immunthrombozytopenie sind sowohl der Thrombozytenabbau beschleunigt als auch die Neubildung gestört. Dadurch entsteht eine Blutungsneigung.

Das Akronym ITP steht für Immunthrombozytopenie und bezeichnet eine immunologisch vermittelte, isolierte Thrombozytopenie, bei der Antikörper gegen die körpereigenen Thrombozyten gebildet werden. Auch wenn man heute weiß, dass bei der ITP neben immunologischen noch andere pathophysiologische Mechanismen eine Rolle spielen, hat sich der Begriff Immunthrombozytopenie durchgesetzt. Die Bezeichnung idiopathische thrombozytopenische Purpura sollte nicht mehr verwendet werden [5]. Ein weit verbreitetes Eponym ist der Begriff Morbus Werlhof.
Bei der ITP unterscheidet man

- eine primäre ITP, ohne erkennbare Ursache,

- von der sekundären ITP, die im Rahmen anderer Erkrankungen auftritt (Tab. 2).

Es werden 3 Krankheitsphasen unterschieden:

- akute ITP (bis zum 3. Monat),

- persistierende ITP (3-12 Monate),

- chronische ITP (länger als 12 Monate).

Für die Erstdiagnose einer ITP sollte die Thrombozytenzahl $<100000 / \mu$ l liegen [5].

Bei der ITP unterscheidet man eine primäre Form, in der sich keine andere Grunderkrankung als Ursache der ITP feststellen lässt, von sekundären Formen, wenn die ITP von Medikamenten ausgelöst wird oder im Rahmen anderer Erkrankungen auftritt (Tab. 2) [6].

Die akute ITP ist der typische Verlauf im Kindesalter $(<10$ Jahre); in der Regel bildet sich die Thrombozytopenie auch ohne Therapie innerhalb weniger Wochen vollständig zurück. Im Erwachsenenalter verläuft die ITP dagegen häufig chronisch und zum Teil über Jahre.

Bisher wurde die akute von der chronischen ITP allein aufgrund einer Erkrankungsdauer über 6 Monate unterschieden, weil bei Erwachsenen Spontanremissionen nach 6 Monaten selten sind. Heute weiß man, dass bei Erwachsenen Spontanremissionen auch noch 12 Monate nach Diagnosestellung vorkommen können [7, 8], danach sind sie jedoch außerordentlich selten [9]. Deshalb wurde kürzlich eine neue Einteilung in 
Tab. 1. Pathophysiologische Einteilung der Thrombozytopenien

\begin{tabular}{l}
\hline Verminderte Bildung \\
\hline Knochenmarkstoxizität (Medikamente, \\
Alkohol, Zytostatika und andere) \\
Infiltration des Knochenmarks (hämatol. \\
Neoplasien, seltener solide Tumore) \\
Myelodysplastische Syndrome \\
Knochenmarkshypoplasie/-aplasie \\
Knochenmarksfibrose \\
Schwerer Vitamin-, Eisenmangel \\
Seltene genetische Defekte: \\
Bernard-Soulier-Syndrom, Glanzmann- \\
Thrombasthenia und andere \\
Neu: ITP mit relativer Bildungsstörung \\
\hline
\end{tabular}

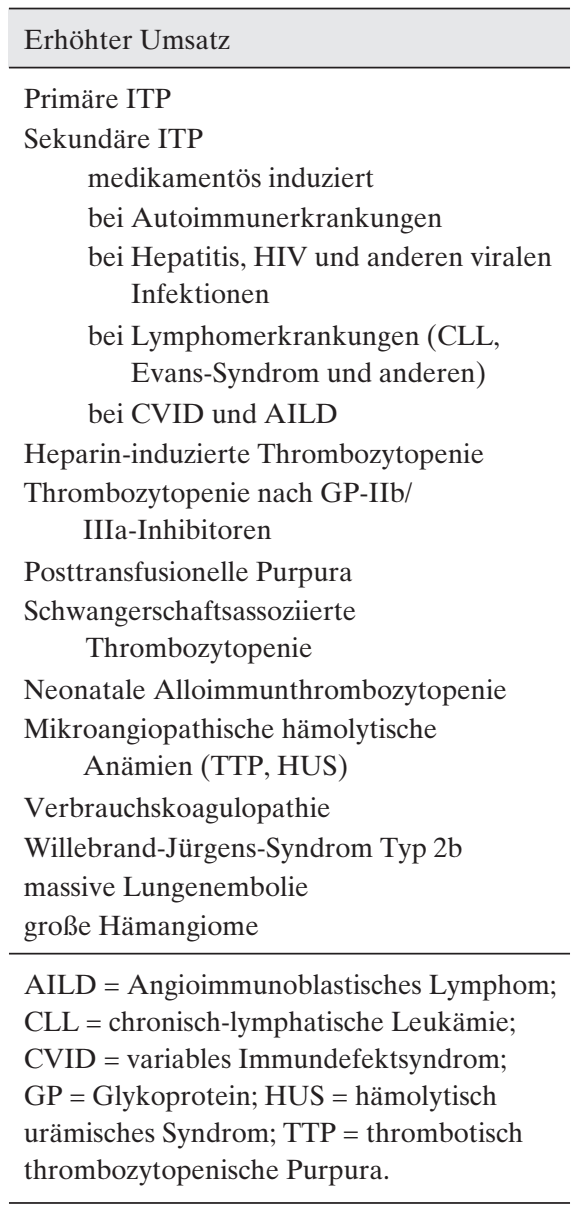

\section{Andere}

Verteilungsstörung bei Splenomegalie

Thrombozytenverlust bei massiver Blutung

Pseudothrombozytopenie (EDTA-Thrombozytopenie)
Tab. 2. Einteilung der Immunthrombozytopenien

\begin{tabular}{ll}
\hline Art der Immunthrombozytopenie & Ursache \\
\hline Primäre ITP & keine auslösende Ursache erkennbar \\
Sekundäre ITP & Medikamentenreaktion \\
& Autoimmunerkrankung (SLE, rheumatoide Arthritis \\
$\quad$ und andere) & Antiphospholipid-Syndrom \\
& CVID \\
& Lymphom (CLL, Hodgkins und andere) \\
& Evans-Syndrom \\
& autoimmun-lymphoproliferatives Syndrom \\
& Infektionen (HIV, Hepatitis B, Hepatitis C, H. pylori) \\
& nach Impfung \\
& andere \\
Thrombozytopenie bei GP-IIb/IIIa-Inhibitortherapie & HIT \\
posttransfusionelle Purpura \\
schwangerschaftsassoziierte Thrombozytopenie \\
neonatale Alloimmunthrombozytopenie
\end{tabular}

CLL = Chronisch-lymphatische Leukämie CVID = variables Immundefektsyndrom; GP = Glykoprotein; HIT = Heparininduzierte Thrombozytopenie; HIV = humanes Immuninsuffizienz-Virus; SLE = systemischer Lupus erythematodes. 
3 Krankheits- und Therapiephasen [5] vorgeschlagen und von den aktuellen internationalen Konsensusleitlinien übernommen [4]:

- Neu-diagnostizierte ITP: bis zu 3 Monate nach Diagnosestellung, Zeitraum der $1^{\text {st }}$-Line-Therapie.

- Persistierende ITP: zwischen 3 und 12 Monaten nach Diagnosestellung, Zeitraum der $2^{\text {nd }}$-Line-Therapie.

- Chronische ITP: mehr als 12 Monate nach Diagnosestellung. Eine spontane Remission oder das Erreichen einer kompletten Remission mit einer weiteren medikamentösen Therapie ist jetzt nicht mehr sehr wahrscheinlich.

Im Folgenden soll ausschließlich die Diagnostik und Therapie der primären ITP im Erwachsenenalter dargestellt werden. Eine ausführliche Besprechung der sekundären ITP-Formen, der pädiatrischen ITP und der ITP bei Schwangeren übersteigt den Umfang dieser Leitlinie. Es wird auf die entsprechenden Publikationen der deutschen und internationalen Fachgesellschaften verwiesen [2, 4, 10-12].

\section{Epidemiologie}

Die ITP ist eine erworbene Erkrankung. Die Inzidenz liegt bei 0,2-0,4 Neuerkrankungen pro 10 000/Jahr und die Prävalenz bei ca. 2 chronischen ITP-Patienten pro 10000 [13-18]. Das sind weniger als 5 Patienten pro 10000 Einwohner; damit gilt die ITP als seltene Erkrankung (Orphan Disease).

\section{Pathophysiologie}

\section{Vermehrter Thrombozytenabbau}

Die ITP ist eine Autoimmunerkrankung. Bei $60-80 \%$ der Patienten findet man mit modernen Untersuchungsmethoden Autoantikörper gegen Epitope zumeist auf den Glykoproteinrezeptoren Ib/IX (von-Willebrand-Faktor-Rezeptor, Thrombinrezeptor) und IIb/IIIa (Rezeptor für Fibrinogen, von-Willebrand-Faktor und andere) [19] und Glykoprotein V. Die antikörperbeladenen Thrombozyten werden von Makrophagen und dendritischen Zellen z.B. in der Milz aufgenommen und abgebaut. Studien der letzten Jahre zeigen, dass neben den Antikörper produzierenden B-Lymphozyten auch Störungen der sogenannten «regulatorischen» T-Lymphozyten eine Rolle spielen [20-23].

In den allermeisten Fällen bleibt unklar, was bei der ITP die Immunreaktion gegen die Thrombozyten in Gang gesetzt hat [24]. Nur bei wenigen Patienten können Medikamente, Infektionen (Hepatitis C, humanes Immundefizienz-Virus (HIV), Helicobacter pylori), eine hämatologische (Lymphome) oder eine andere Erkrankung (systemischer Lupus erythematodes (SLE), rheumatoide Arthritis und andere) als Auslöser erkannt werden [6, 25], die dann als sekundäre ITP abzugrenzen sind (Tab. 2).

\section{Verminderte Thrombozytopoese}

Bisher ging man davon aus, dass bei der ITP sowohl der Thrombozytenabbau als auch die Neubildung von Thrombozyten erhöht sind. Ein Teil der Patienten hat jedoch zusätzlich zum vermehrten Thrombozytenabbau eine insuffiziente Thrombozytopoese [26-28]. Möglicherweise stören die Antikörper gegen Thrombozyten-Glykoproteine auch die Reifung der Megakaryozyten im Knochenmark [29-33].

Darüber hinaus gibt es noch mindestens einen weiteren Mechanismus. Bei ITP-Patienten sind die Thrombopoietinspiegel im Vergleich zu gesunden Menschen zwar erhöht, aber dennoch deutlich niedriger als bei anderen Erkrankungen, die einen vergleichbaren Thrombozytenmangel aufweisen (z.B. aplastische Anämie oder auch nach Chemotherapie). Man spricht deshalb von einem relativen Thrombopoietinmangel $[34,35]$. Die Ursache für den relativen Thrombopoietinmangel liegt nach derzeitiger Vorstellung in einem gesteigerten Abbau von Thrombopoietin. Thrombopoietin wird physiologischerweise an Thrombozyten gebunden und zusammen mit diesen Thrombozyten in der Milz phagozytiert und zerstört [35]. Dies hat zur Entwicklung der TRAs und zu neuen Therapiekonzepten geführt.

\section{Prävention}

Eine Strategie zur Prävention der ITP ist nicht bekannt.

\section{Stadieneinteilung}

Der Schweregrad einer ITP und die sich daraus ergebenden diagnostischen und therapeutischen Konsequenzen orientieren sich primär an der klinischen Blutungsneigung und nicht an der Thrombozytenzahl.

In den bisherigen Leitlinien orientierten sich Diagnostik- und Therapieempfehlungen primär an der Thrombozytenzahl [1, 2]. Es ist die Meinung des Expertenpanels, dass weder die Diagnostik noch die Therapie oder die Risikoprognose von ITP-Patienten an der Thrombozytenzahl festgemacht werden dürfen. Im Vordergrund steht immer die klinische Blutungsneigung.

Für Kinder [36, 37] und für Therapiestudien [38, 39] wurden zahlreiche Blutungsscores entwickelt, die sich in der Praxis bisher nicht durchgesetzt haben. Auch die neue Einteilung in Blutungsschweregrade der internationalen Konsensusleitlinie [4] erscheint für die klinische Anwendung bei Erwachsenen wenig geeignet. Die Beurteilung des klinischen Schweregrades sollte nach Meinung der Autoren weiter nach dem etablierten Blutungsscore der Weltgesundheitsorganisation (World Health Organisation (WHO)) erfolgen (Tab. 3). 
Tab. 3. Blutungsgrade analog WHO und NCI CTCAE (MedDRA v9.0) [165-167]

\begin{tabular}{|c|c|}
\hline WHO Blutungsgrad & CTCAE \\
\hline 0 & keine Blutungszeichen \\
\hline I & $\begin{array}{l}\text { Petechien } \\
\text { kleine Hämatome, Ekchymosen }(<10 \mathrm{~cm}) \\
\text { Schleimhautblutungen (Mund, Nase) } \\
\text { Epistaxis (<1 h Dauer, keine ärztliche Intervention notwendig) } \\
\text { subconjunctivale Blutungen } \\
\text { vaginale Blutungen (unabhängig von Menstruation, nicht mehr als } 2 \text { Binden/Tag notwendig) }\end{array}$ \\
\hline II (keine Transfusion notwendig) & $\begin{array}{l}\text { Hämatome, Ekchymosen (> } 10 \mathrm{~cm} \text { Durchmesser) } \\
\text { Epistaxis (> } 1 \text { h Dauer oder Tamponade notwendig) } \\
\text { retinale Blutungen ohne Visusverminderung } \\
\text { vaginale Blutungen (unabhängig von Menstruation, mehr als } 2 \text { Binden/Tag notwendig) } \\
\text { Melaena, Hämatemesis, Hämoptysen, Hämaturie, Hämatochezie } \\
\text { Blutungen aus Punktionsstellen } \\
\text { Blutungen in Muskeln und Gelenke }\end{array}$ \\
\hline III (transfusionspflichtige Blutungen) & $\begin{array}{l}\text { Epistaxis } \\
\text { Schleimhautblutungen (Mund, Nase) } \\
\text { vaginale Blutungen (unabhängig von Menstruation) } \\
\text { Melaena, Hämatemesis, Hämoptysen, Hämaturie, Hämatochezie } \\
\text { Blutungen aus Punktionsstellen } \\
\text { Blutungen in Muskeln und Gelenke }\end{array}$ \\
\hline IV (unabhängig vom Transfusionsbedarf) & $\begin{array}{l}\text { retinale Blutungen mit Visusverminderung } \\
\text { ZNS-Blutungen } \\
\text { andere Organblutungen, die die Funktion der betroffenen Organe (Gelenke, Muskulatur, Niere, } \\
\quad \text { Lunge usw.) gefährden } \\
\text { Letale Blutungen (in den NCI CTCAE auch als Blutungsgrad V bezeichnet) }\end{array}$ \\
\hline
\end{tabular}

CTCAE = Common terminology criteria for adverse events; $\mathrm{NCI}=$ National Cancer Institute; $\mathrm{WHO}=$ Weltgesundheitsorganisation; $\mathrm{ZNS}=$ Zentralnervensystem.

\section{Prognose}

Bei der chronischen ITP des Erwachsenen sind spontane Remissionen selten (5-9\%) [1, 7]. Bei therapierefraktärer Thrombozytopenie steigt das Risiko für fatale Blutungen mit dem Lebensalter von 1-4\%/Jahr bei jungen Menschen [40] auf fast $13 \% / J a h r$ bei über Sechzigjährigen [41]. Neben Blutungen sind Infektionen, wahrscheinlich bedingt durch die immunsuppressive Therapie, eine ebenso wichtige Todesursache [42]. Es ist die Erfahrung der Panelmitglieder, dass ITP-Patienten mit therapierefraktärer Thrombozytopenie und wiederholten leichtergradigen Blutungen ein höheres Risiko tragen, im weiteren Verlauf auch schwere oder tödliche Blutungen zu entwickeln, während andere ITP-Patienten ohne klinische Blutungsneigung einen jahrelangen problemlosen Krankheitsverlauf haben.

\section{Klinik}

Bei fast einem Drittel der Patienten sind überhaupt keine Symptome erkennbar [15]. Zu den typischen Blutungszeichen gehören:
- Petechien an den abhängigen Körperpartien,

- Schleimhautblutungen,

- verstärkte Blutungen bei geringen Verletzungen,

- verstärkte Periodenblutungen,

- urogenitale Blutungen,

- Hämatomneigung schon bei kleinen Traumen,

- Anämie durch chronischen Blutverlust und sekundären Eisenmangel.

\section{Diagnostik}

Die Diagnostik bei der Erstvorstellung eines thrombozytopenen Patienten (sogenannte Basisdiagnostik), bei dem die Diagnose ITP bisher nicht gesichert ist, aber vermutet wird, unterscheidet sich naturgemäß von der Diagnostik bei Patienten mit bekannter persistierender oder chronischer ITP, bei denen andere therapeutische Entscheidungen getroffen werden müssen. 
Basisdiagnostik bei Erstvorstellung eines Patienten mit isolierter Thrombozytopenie

Die Basisdiagnostik sollte auf einige wenige und einfach zu erhebende Befunde begrenzt werden:

- Anamnese: aktuelle und frühere Blutungen, Medikamente, Infektionen, Alkohol, Schwangerschaft, frühere Thrombosen, Familienanamnese, Berufsanamnese;

- Körperliche Untersuchung: Blutungszeichen, Lymphknoten, Leber-, Milzgröße, usw.;

- Blutbild: EDTA und Citrat, zum Ausschluss einer Pseudothrombozytopenie;

- Begutachtung des Blutausstriches durch einen in der Diagnostik von Thrombozytopenien erfahrenen Arzt;

- Gerinnungsparameter: Thromboplastinzeit (Quick-Wert), aktivierte partielle Thromboplastinzeit (aPTT);

- Blutgruppe und irreguläre erythrozytäre Autoantikörper, insbesondere bei gleichzeitiger Anämie;

- Knochenmarksdiagnostik nur bei atypischen Befunden und bei älteren Patienten ( $>60$ Jahre).

Bei unauffälliger Anamnese ist der Nachweis einer isolierten Thrombozytopenie bei normalen übrigen hämatologischen Parametern (Erythrozyten-, Leukozytenzahl, Differenzialblutbild, Gerinnungswerte) zunächst ausreichend für die Diagnose ITP. Dazu gehört immer die umgehende Begutachtung des Blutausstriches durch einen in der Diagnostik von Thrombozytopenien erfahrenen Arzt (eine thrombotisch thrombozytopenische Purpura (TTP) ist eine hämatologische Notfalldiagnose und darf nicht übersehen werden!).

Die Bestimmung der Thrombozytenzahl in Citrat- oder Heparin-Blut zum Ausschluss einer Pseudothrombozytopenie (Synonym: EDTA-Thrombozytopenie) ist obligater Bestandteil der initialen Diagnostik. Diese kann bereits im Blutausstrich (s.o.) erkannt werden.

Die nächste häufige Ursache einer Thrombozytopenie sind Medikamente. Es ist die Erfahrung der Panelmitglieder, dass Patienten nichtverschreibungspflichtige Präparate (naturheilkundliche Wirkstoffe, Schmerztabletten, Vitamine, «Aufbaupräparate» und andere) häufig nicht als Medikamente bewerten und nur auf gezielte Nachfrage nennen; dies gilt auch für Alkohol. Eine Liste der häufigsten Thrombozytopenie auslösenden Medikamente findet sich unter http://w3.ouhsc.edu/ platelets/index.html (überprüft 2/2010).

Bei jedem blutenden Patienten wird man im Rahmen der Basisdiagnostik auch Gerinnungsparameter (Prothrombinzeit $(\mathrm{PT})=$ Quick-Wert, aPTT) bestimmen. Veränderungen von PT und aPTT sind nicht typisch für die ITP und sprechen für alternative Diagnosen (Verbrauchskoagulopathie, Lebersynthesestörung, Antiphospholipid-Syndrom und andere).

Die Bestimmung von Blutgruppe und erythrozytären Autoantikörpern wird als notwendiger Teil der Basisdiagnostik betrachtet, wenn der Patient eine Anämie hat, einerseits um auf eine eventuelle Transfusion besser vorbereitet zu sein, andererseits um ein Evans-Syndrom auszuschließen. Bis zu 25\% der ITP-Patienten haben einen positiven direkten Antiglobulin-Test [43]. Das gleichzeitige Vorliegen einer Thrombozytopenie und eines positiven direkten Antiglobulintests unterstützt die Differenzialdiagnose, dass auch die Thrombozytopenie immunologisch bedingt ist. Die Kombination von autoimmunhämolytischer Anämie und autoimmuner Thrombopenie bezeichnet man als Evans-Syndrom. Bei einem Drittel der Patienten mit Evans-Syndrom kann die Thrombozytopenie der Anämie vorausgehen und als primäre ITP zunächst verkannt werden. Außerdem wären bei einem Evans-Syndrom Anti-D-Präparate kontraindiziert. Bei einem positiven direkten Antiglobulin-Test sollte deshalb die Diagnostik erweitert werden (Sonografie, Röntgen-Thorax, Knochenmarkspunktion).

\section{Ist eine Knochenmarkspunktion im Rahmen der Erstdiagnostik notwendig?}

In der Regel ist bei sonst typischer Anamnese und Befunden durch die Knochenmarkspunktion keine Änderung der Diagnose zu erwarten. Andererseits beschreiben neuere Studien, dass bei genauerer Untersuchung 3-10\% der ITP-Diagnosen revidiert werden müssen $[18,44]$, insbesondere bei therapierefraktärer ITP [45]. Es ist die Meinung der Panelmitglieder, dass eine Knochenmarksdiagnostik nur in Ausnahmefällen zur Basisdiagnostik gehören sollte:

- bei anamnestischen oder körperlichen Untersuchungsbefunden, die nicht typisch für eine ITP sind (z.B. vergrößerte Lymphknoten, Leber-/Milzvergrösserung);

- wenn neben der Thrombozytenzahl auch andere Laborwerte, insbesondere Leukozyten- und Erythrozytenparameter verändert sind;

- bei Patienten > 60 Jahre wegen der zunehmenden Häufigkeit alternativer Diagnosen: Lymphome, myelodysplastische Syndrome, Plasmozytom und andere.

\section{Weiterführende Diagnostik bei persistierender oder chronischer ITP}

Bei bekannter ITP ist eine Ausweitung der Diagnostik sinnvoll bei

- Patienten, deren Thrombozytenwerte unter der $1^{\text {st }}$-LineTherapie (zumeist Steroide) gar nicht oder nur minimal ansteigen,

- Patienten, deren Thrombozytenzahl auf die SteroidTherapie zunächst anspricht, die bei der notwendigen Dosisreduktion des Steroids dann aber einen erneuten Thrombozytenabfall entwickeln.

Es ist die Erfahrung der Panelmitglieder, dass Patienten trotz gar nicht oder nur minimal ansteigender Thrombozytenwerte zum Teil 4 Wochen und länger mit hochdosierten Steroiden behandelt werden. Bevor bei persistierender oder chronischer ITP langfristige und zum Teil belastende Therapieversuche 
Tab. 4. Diagnostik und Differenzialdiagnostik bei Verdacht auf ITP

\begin{tabular}{|c|c|}
\hline Differenzialdiagnose & Anamnese, typische Befunde \\
\hline Pseudothrombozytopenie (EDTA-Thrombozytopenie) & $\begin{array}{l}\text { häufigste Differenzialdiagnose; Bestimmung der Thrombozytenzahl } \\
\text { in Citrat oder Heparin }\end{array}$ \\
\hline Hereditäre Thrombozytopenie & $\begin{array}{l}\text { Familienanamnese, Untersuchung des Blutausstriches und MPV, } \\
\text { ob sehr große (Makrothrombozytopenie) oder kleine Thrombozyten } \\
\text { (Wiskott-Aldrich-Syndrom), usw. }\end{array}$ \\
\hline Medikamenten-induzierte Thrombozytopenie & $\begin{array}{l}\text { Anamnese, Testung auf medikamentenabhängige } \\
\text { Thrombozyten-Antikörper }\end{array}$ \\
\hline Zytostatika-induzierte Thrombozytopenie & Anamnese \\
\hline Virustatika-induzierte Thrombozytopenie & Anamnese \\
\hline Heparin-induzierte Thrombozytopenie & Anamnese \\
\hline Posttransfusionelle Purpura & kurz zurückliegende Bluttransfusionen \\
\hline Schwangerschaftsassoziierte Thrombozytopenie & nur schwangere Patientinnen \\
\hline Lymphom & $\begin{array}{l}\text { Anamnese, B-Symptome, Sonografie der Lymphknotenstationen, } \\
\text { Milzgröße, eventuell Knochenmarkspunktion }\end{array}$ \\
\hline Infektionen (Viren, Bakterien, Parasiten) & $\begin{array}{l}\text { Serologie auf HIV, CMV, EBV, Röteln, Parvovirus B19, Hantaviren } \\
\text { und andere bakteriologische Untersuchungen, Blutkultur bei Verdacht } \\
\text { auf Sepsis, Blutausstrich, dicker Tropfen bei Verdacht auf Malaria }\end{array}$ \\
\hline Lebererkrankungen & $\begin{array}{l}\text { Leberwerte, Hepatitis-Serologie, Sonografie ob Splenomegalie, } \\
\text { Gaucher-Diagnostik }\end{array}$ \\
\hline Alkoholabusus & muss häufig gezielt erfragt werden \\
\hline Sarkoidose & pneumologische Diagnostik \\
\hline Schwere Vitaminmängel (B12, Folsäure, auch schwerster Eisenmangel) & Labordiagnostik \\
\hline Autoimmunerkrankungen & $\begin{array}{l}\text { Labordiagnostik für Lupus erythematodes, rheumatoide Arthritis, } \\
\text { Antiphospholipid-Syndrom, Autoimmunthyreoiditis und andere } \\
\text { Autoimmunsyndrome }\end{array}$ \\
\hline Evans-Syndrom & Anämie, positiver direkter Antiglobulin-Test \\
\hline $\begin{array}{l}\text { Hämatologische Systemerkrankungen (akute Leukämie, myeloprolife- } \\
\text { rative Syndrome, Myelodysplasie, Plasmozytom, Lymphome, CVID, } \\
\text { autoimmun-lymphoproliferatives Syndrom, aplastische Anämie, } \\
\text { paroxysmale nächtliche Hämoglobinurie, Graft-versus-Host-Erkrankung) }\end{array}$ & $\begin{array}{l}\text { Veränderung auch anderer Blutzellreihen, der Serum-Immunglobuline, } \\
\text { Knochenmarkspunktion eventuell mit Durchflusszytometrie und } \\
\text { Zytogenetik }\end{array}$ \\
\hline $\begin{array}{l}\text { Thrombotisch thrombozytopenische Purpura und hämolytisch } \\
\text { urämisches Syndrom }\end{array}$ & $\begin{array}{l}\text { meist weitere Symptome: Fieber, Hämolyse, Niereninsuffizienz, } \\
\text { neurologische Symptome usw. }\end{array}$ \\
\hline Verbrauchskoagulopathie & Veränderung weiterer Gerinnungsparameter \\
\hline Große Hämangiome (z.B. Kasabach-Merritt-Syndrom) & klinisches Bild \\
\hline
\end{tabular}

$\mathrm{CMV}=$ Cytomegalovirus; CVID = variables Immundefektsyndrom; EBV = Epstein-Barr-Virus; HIV = humanes Immuninsuffizienz-Virus;

$\mathrm{MPV}=$ mittleres Plättchenvolumen.

unternommen werden, sollten deshalb andere Thrombozytopenieursachen differenzialdiagnostisch ausgeschlossen werden (Tab. 4). Insbesondere wenn die Thrombozyten unter der $1^{\text {st }}$-Line-Therapie gar nicht oder nur minimal angestiegen sind, muss eine alternative hämatologische oder immunologische Ursache durch entsprechende Diagnostik inklusive Knochenmarkspunktion ausgeschlossen werden (Tab. 5).

Patienten mit seltenen hereditären oder erworbenen Thrombozytopenien (Wiskott-Aldrich-Syndrom, Bernard-Soulier-Syndrom, Makrothrombozytopenie bei MYH9-Mutationen und andere) werden nicht selten als chronische ITP verkannt und zum Teil über Jahre einer Vielzahl von letztlich unwirksamen Therapien unterzogen. Der Parameter mittleres Plättchenvolumen (MPV; Normbereich 7-12 fl), der von fast allen modernen Hämatologieautomaten zusätzlich $\mathrm{zu}$ den Basisparametern automatisch mitbestimmt wird, kann hier differenzialdiagnostisch hilfreich sein. Bei einer ITP ist das MPV häufig erhöht. Ungewöhnlich wären jedoch ein sehr hohes MPV (> 12,4 fl, dann MYH9-Mutationen oder Bernard-Soulier-Syndrom erwägen) [46] oder ein niedrig-normales MPV (häufig bei Wiskott-Aldrich-Syndrom). Da einzelne Laborgeräte aber bei der Erkennung und Zählung von sehr großen oder sehr kleinen Thrombozyten Probleme haben, sollten diese Differenzialdiagnosen immer durch eine zusätzliche Begutachtung des Blutausstriches von einem in der Diagnostik von Thrombozytopenien erfahrenen Arzt ausgeschlossen werden.

Eine HIV-Erkrankung sowie Hepatitis B und C können initial durch eine isolierte Thrombozytopenie auffallen. Deshalb sollten sie vor der Gabe immunsuppressiver Medikamente (z.B. Rituximab, Azathioprin und anderen) ausgeschlossen werden.

\section{Helicobacter pylori - Diagnostik und Therapie}

Eine H. pylori Infektion kann in Einzelfällen das Auftreten oder Persistieren einer ITP begünstigen. Durch neue, nichtin- 
Tab. 5. Diagnostik bei persistierender und chronischer ITP

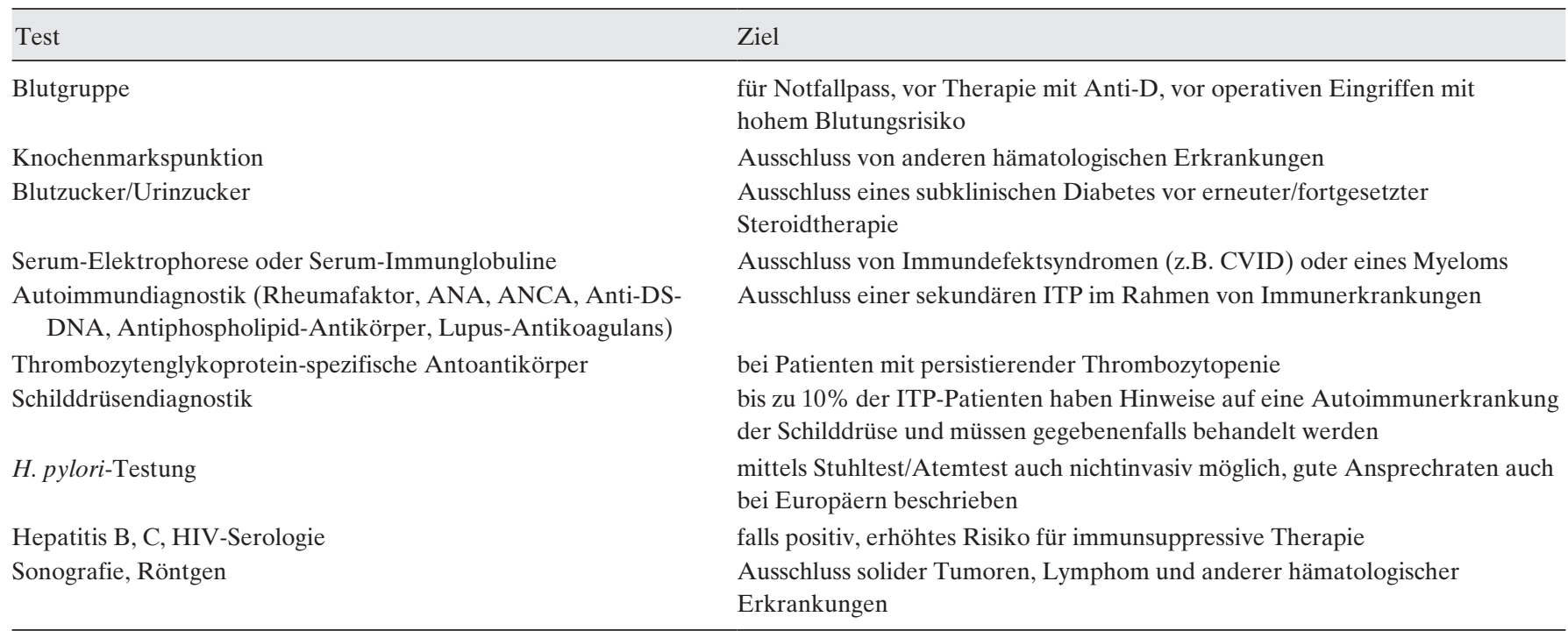

ANA = Antinukleäre Antikörper; ANCA = Anti-Neutrophile cytoplasmatische Antikörper; CVID = variables Immundefektsyndrom;

DS = doppelsträngig; HIV = humanes Immuninsuffizienz-Virus.

vasive Untersuchungsmethoden (Stuhltest/Atemtest) kann $H$. pylori auch ohne Biopsie der Magenschleimhaut nachgewiesen werden. Die Erfolgsraten einer Eradikationstherapie sind bei asiatischen Patienten besser als bei Europäern [47-52]. Angesichts der Einfachheit der Diagnostik und der niedrigen Kosten einer H. pylori-Eradikation ist diese dennoch sinnvoll.

\section{Untersuchung auf Thrombozyten-Antikörper}

Thrombozytenglykoprotein-spezifische Antoantikörper lassen sich je nach verwendeter Methode bei $60-80 \%$ der Patienten nachweisen [19]. Dabei sollte nicht nur der Nachweis freier Antikörper im Plasma/Serum, sondern insbesondere auch der von Antikörpern auf den zirkulierenden Patiententhrombozyten angestrebt werden (s.u.). Ein positiver Nachweis mit einem hoch sensitiven und spezifischen Test (Monoklonaler Antikörper-Immobilisationstest für plättchenspezifische Antigene, MAIPA, s.u.) belegt, dass eine Thrombozytopenie immunologisch bedingt ist, differenziert dabei aber nicht zwischen primärer und sekundärer ITP.

Bei Patienten mit einer akuten, postinfektiösen Thrombozytopenie, deren Thrombozytenzahlen sich rasch wieder erholen, ist die Untersuchung auf Thrombozyten-Autoantikörper nicht sinnvoll.

Bei Patienten mit Persistenz der Thrombozytopenie erlaubt der Nachweis der Antikörper die Diagnose zu sichern bzw. andere Differentialdiagnosen weitestgehend auszuschließen. Dies gilt insbesondere für Patienten mit atypischen Befunden (Liste nicht vollständig):

- fehlende Ansprache auf Steroide (hilft eine längere, letztlich unwirksame Steroidtherapie zu vermeiden),

- fehlende Ansprache auf intravenöse (i.v.) Immunglobuline oder Anti-D,
- Verdacht auf Alkohol oder medikamentös-toxische Knochenmarksschädigung,

- Verdacht auf hereditäre Thrombozytopenie,

- Lebererkrankung,

- Splenomegalie.

Beim Wiskott-Aldrich-Syndrom, einer seltenen hereditären Thrombopenie mit assoziierten immunologischen Veränderungen, können in manchen Fällen auch Thrombozyten-Antikörper nachgewiesen werden.

Wenn dagegen Thrombozyten-Antikörper nicht nachgewiesen werden, widerlegt dies nicht die Diagnose ITP. Wenn man sich zur Bestimmung von Thrombozyten-Antikörpern entschließt, dann sind nur Tests auf Glykoprotein-spezifische (Glykoproteine Ib/IX, Glykoprotein IIb/IIIa, Glykoprotein V) thrombozytengebundene Antikörper (z.B. MAIPA) ausreichend sensitiv und spezifisch. Die Präanalytik (Wie viel Blut? Welches Antikoagulans? Transportzeit?) sollte mit dem Labor abgestimmt sein, um Fehlbestimmungen zu vermeiden. Der Nachweis freier Antikörper im Plasma/Serum hat eine Sensitivität von nur $\sim 20 \%$ [53]. Andere Testverfahren (plättchenassoziierte Immunglobuline und andere) führen häufig zu falsch-positiven Befunden und sollen nicht mehr eingesetzt werden [4].

\section{Wann ist eine Krankenhauseinweisung bei ITP-Patienten gerechtfertigt?}

Die Entscheidung, einen Patienten zur Überwachung und Einleitung weiterer therapeutischer Maßnahmen stationär einzuweisen, orientiert sich allein an der klinischen Blutungssituation, nicht an der Thrombozytenzahl. 
Tab. 6. Therapiesequenz bei ITP

\begin{tabular}{|c|c|c|}
\hline Therapielinie & Bisher & Neu \\
\hline $1^{\text {st }}$-Line & $\begin{array}{l}\text { Steroide bei starker Blutung }\left({ }^{\circ} \mathrm{III},{ }^{\circ} \mathrm{IV}\right) \text { zusätzlich i.v. Immunglobuline } \\
\text { (eventuell Anti-D), eventuell Thrombozytenkonzentrate }\end{array}$ & wie bisher \\
\hline $2^{\text {nd }}$-Line & Azathioprin, Vinca-Alkaloide oder Splenektomie & TRA $^{\mathrm{a}}$, Rituximab oder wie bisher \\
\hline $3^{\text {rd }}$-Line & Azathioprin, Vinca-Alkaloide & TRA $^{\mathrm{a}}$, Rituximab oder wie bisher \\
\hline Post $3^{\text {rd }}$-Line & $\begin{array}{l}\text { Anti-D, Cyclophosphamid, Cyclosporin, Mycophenolat } \\
\text { mofetil, Danazol, Dapson, Alemtuzumab, Kombinations- } \\
\text { chemotherapie, Stammzelltransplantation }\end{array}$ & wie bisher \\
\hline
\end{tabular}

Tab. 7. Absolute und relative Kontraindikationen für eine Steroidtherapie

\begin{tabular}{|c|c|}
\hline \multirow[t]{2}{*}{$\begin{array}{l}\text { Absolute Kontraindikationen für eine } \\
\text { Steroidtherapie }\end{array}$} & $\begin{array}{l}\text { akute Virusinfektionen (z.B. Herpes simplex, } \\
\text { Herpes zoster, Varizellen und andere) }\end{array}$ \\
\hline & HBsAG-positive, chronisch aktive Hepatitis \\
\hline \multirow{11}{*}{$\begin{array}{l}\text { Relative Kontraindikationen für eine } \\
\text { Steroidtherapie }\end{array}$} & Magen- oder Darmgeschwüre \\
\hline & akute und chronische bakterielle Infektionen \\
\hline & Tuberkulose in der Anamnese \\
\hline & akute und chronische systemische Mykosen \\
\hline & Parasitosen \\
\hline & schwer einstellbarer Bluthochdruck \\
\hline & Diabetes mellitus \\
\hline & Osteoporose \\
\hline & Psychose in der Anamnese \\
\hline & Eng- und Weitwinkelglaukom \\
\hline & Divertikulitis und frische Enteroanastomosen \\
\hline
\end{tabular}

Eine Thrombozytopenie bei gesicherter ITP rechtfertigt für sich alleine auch bei sehr niedrigen Werten keine stationäre Einweisung. Ein in der Behandlung von ITP-Patienten erfahrener Arzt, in der Regel ist das ein Hämatologe, Hämostaseologe oder Transfusionsmediziner, sollte immer in die Entscheidung mit eingebunden werden. Bei chronischen ITP-Patienten, die zum Teil einen sehr individuellen und vom «Regelfall» abweichenden Krankheitsverlauf haben, sollte außerdem versucht werden, den für die bisherige Betreuung dieses ITP-Patienten verantwortlichen Arzt oder die Klinik/Fachpraxis zu kontaktieren. Es wird empfohlen, dass der Patient einen ITP-Pass oder ein anderes Dokument mit den notwendigen Kontaktdaten erhält, möglichst auch mit Informationen zum bisherigen Krankheitsverlauf (Blutungsereignisse, Ansprechen auf Therapie, Therapiekomplikationen).

\section{Therapie}

Einen Überblick über die Therapiesequenz bei ITP gibt die Tabelle 6.

\section{$1^{\text {st }}$-Line-Therapie der neu diagnostizierten ITP}

Die Indikation zur $1^{\text {st }}$-Line-Therapie orientiert sich primär an der Blutungsneigung.

\section{Neu diagnostizierte ITP mit Blutungsneigung}

Bei allen Patienten mit erstmalig diagnostizierter ITP und WHO Grad-III- oder Grad-IV-Blutungen (Tab. 3) besteht eine Therapieindikation unabhängig von der Thrombozytenzahl. Als $1^{\text {st }}$-Line-Therapie soll eine Behandlung mit Corticosteroiden angeboten werden, außer es bestehen Kontraindikationen gegen Corticosteroide (Tab. 7). Die hohe Steroiddosis sollte nach 1-2 Wochen reduziert und dann ausgeschlichen werden, eine maximale Therapiedauer von 2-3 Monaten sollte nicht überschritten werden. Insbesondere wenn sich unter der Therapie die Blutungssymptomatik nicht deutlich bessert, sollte die hochdosierte Steroidbehandlung nicht lange fortgesetzt, sondern rasch beendet werden. Eine Dauertherapie unter der Cushing-Schwelle (z.B. unter 5 mg Prednison/ Tag) und bei genauer Kontrolle auf Nebenwirkungen (Osteoporose usw.) ist in Einzelfällen möglich. 
Tab. 8. Häufig eingesetzte Medikamente und Dosierungen bei ITP

\begin{tabular}{|c|c|}
\hline Predniso(lo)n & $\begin{array}{l}\text { 1-4 mg/kg p.o. tgl. für } 1-2 \text { Wochen, danach langsame Dosisreduktion, } \\
\text { nach 2-3 Monaten sollte die Steroidtherapie beendet sein }\end{array}$ \\
\hline Dexamethason & 40 mg p.o. tgl., $\times 4$ Tage, $4-6$ Zyklen alle $14-28$ Tage \\
\hline Intravenöse Immunglobuline & $0,4-1 \mathrm{~g} / \mathrm{kg} / \mathrm{Tag}$, eventuell über mehrere Tage \\
\hline Azathioprin & $2 \mathrm{mg} / \mathrm{kg}$ p.o. tgl., Ansprechen zum Teil erst nach Monaten \\
\hline Vincristin & $1-2 \mathrm{mg} 1 \times$ pro Woche, $4-6$ Gaben \\
\hline Vinblastin & 5-10 mg $1 \times$ pro Woche, 4-6 Gaben \\
\hline Romiplostim & $\begin{array}{l}1-10 \mu \mathrm{g} / \mathrm{kg} \text { s.c. } 1 \times \text { pro Woche, Dauertherapie, nach Splenektomie oder } \\
\text { bei Kontraindikationen gegen Splenektomie }\end{array}$ \\
\hline Eltrombopag & $\begin{array}{l}25-75 \text { mg p.o. tgl., Dauertherapie, nach Splenektomie oder bei } \\
\text { Kontraindikationen gegen Splenektomie }\end{array}$ \\
\hline Anti-D ${ }^{a}$ & $\begin{array}{l}50-75 \mu \mathrm{g} / \mathrm{kg} \text { i.v. für } 1-3 \text { Tage (Dosierungsangabe für WinRho }{ }^{\circledR} \text { SDF nach der } \\
\text { amerikanischen «Prescribing Information», Stand 12/2009) }\end{array}$ \\
\hline Rituximab $^{\mathrm{a}}$ & $375 \mathrm{mg} / \mathrm{m}^{2} 1 \times$ pro Woche i.v. für 4 Wochen \\
\hline Cyclophosphamid $^{\text {a }}$ & $1-2 \mathrm{mg} / \mathrm{kg}$ p.o. tgl. \\
\hline Cyclosporin ${ }^{\mathrm{a}}$ & Dosierung nach Blutspiegel, Ziel 100-400 ng/ml \\
\hline Danazol $^{\mathrm{a}}$ & 400-800 mg p.o. tgl. \\
\hline Dapson $^{\mathrm{a}}$ & 75-100 mg p.o. tgl. \\
\hline Myclophenolat $^{\mathrm{a}}$ & $2 \times 250$ bis $2 \times 1000 \mathrm{mg}$ p.o. tgl. \\
\hline Alemtuzumab ${ }^{\mathrm{a}}$ & $10 \mathrm{mg} /$ Tag i.v. für 10 Tage \\
\hline
\end{tabular}

Bei neu diagnostizierten Patienten mit mittelschweren Blutungen (WHO Blutungsgrad II) wird man sich, auch wenn diese Blutungen eigentlich nicht bedrohlich sind, in den meisten Fällen ebenfalls zu einer $1^{\text {st }}$-Line-Therapie entschließen, weil der weitere Verlauf bei diesen Patienten nicht absehbar ist. Eine «Watch-and-Wait»-Strategie ist im Einzelfall aber auch vertretbar.

\section{Neu diagnostizierte ITP mit <30 000 Thrombozyten/ul und geringer oder fehlender Blutungsneigung}

Die Indikation zur $1^{\text {st }}$-Line-Therapie bei Patienten mit erstmalig diagnostizierter ITP und geringer oder gänzlich fehlender Blutungsneigung (WHO Blutungsgrade 0 und I) ist nicht gesichert. Hier müssen Nutzen und Risiken (z.B. berufliches Verletzungsrisiko, Komorbiditäten) individuell abgewogen und mit dem Patienten besprochen werden.

Für die Behandlung asymptomatischer Patienten spricht, dass das Blutungsrisiko bei Thrombozytenzahlen $<30000 / \mu$ l und bei Patienten $>60$ Jahre deutlich erhöht ist $[40,41,54,55]$. Eine Watch-and-Wait-Strategie ist im Einzelfall und insbesondere bei fehlender Blutungsneigung genauso gut vertretbar. Eine über einen initialen Therapieversuch hinausgehende langfristige Steroidbehandlung asymptomatischer Patienten nur mit dem Ziel, die Thrombozytenzahl über $30000 / \mu l$ zu halten, wird abgelehnt.

\section{Neu diagnostizierte ITP mit $>30000$ Thrombozyten/ul und geringer oder fehlender Blutungsneigung}

Patienten mit >30 000 Thrombozyten/ $\mu$ l und ohne Blutungszeichen bedürfen in der Regel keiner Therapie [1, 2,
4]. Andererseits ist ein Therapieversuch nicht kontraindiziert, weil auch diese Patienten durch eine $1^{\text {st }}$-Line-Therapie z.B. mit Steroiden eine anhaltende komplette Remission erreichen können [56-58]. Außerdem wird durch einen Nachweis der Wirksamkeit von Steroiden die Diagnose ITP unterstützt und man kann dem Patienten ein Behandlungsschema für Verletzungen oder Operationen an die Hand geben.

\section{Wahl des Corticosteroids}

Bei der Wahl des Corticosteroids (Prednison, Prednisolon, Dexamethason und andere) gibt es keine Präferenz (Tab. 8).

\section{Notfalltherapie mit i.v. Immunglobulinen}

Die Gabe von i.v. Immunglobulinen ist bei ITP-Patienten mit Grad-III- oder Grad-IV-Blutungen (Tab. 3) indiziert, unabhängig von der Thrombozytenzahl.

Die Gabe von i.v. Immunglobulinen sollte bei blutenden ITP-Patienten mit einer Steroidtherapie kombiniert werden.

Die Gabe von i.v. Immunglobulinen ist bei ITP-Patienten ohne klinische Blutungsneigung auch bei sehr niedrigen Thrombozytenzahlen nicht indiziert.

Die Gabe von i.v. Immunglobulinen vor Operationen ist indiziert, wenn eine Steroidtherapie kontraindiziert oder nicht schnell genug wirksam ist. 
Die Dosis liegt zwischen $0,4 \mathrm{~g} / \mathrm{kg}$ (z.B. über 5 Tage) und $1 \mathrm{~g} / \mathrm{kg}$ (meist 1 oder 2 Tage) [59-61]. Leider hält die Wirkung nicht an und bei fast allen Patienten fällt die Thrombozytenzahl nach 2-4 Wochen wieder ab. Die Nebenwirkungen von i.v. Immunglobulinen sind meist leicht: Kopfschmerzen, Fieber, Knochen- und Muskelschmerzen. Seltene ernste Komplikationen wurden aber auch beschrieben: allergische Reaktionen, Nierenfunktionsstörungen, aseptische Meningitis, Hämolyse, Thromboembolien, Herzinfarkt und zerebrale Durchblutungsstörungen [62-65]. Die Gabe von i.v. Immunglobulinen sollte bei Patienten mit Niereninsuffizienz unter engmaschiger Kontrolle erfolgen. Bei ITP-Patienten, die bekanntermaßen nicht auf i.v. Immunglobuline ansprechen, ist deren Gabe kontraindiziert, ebenso bei Patienten mit hereditärem IgAMangel und Anti-IgA-Antikörpern.

\section{Notfalltherapie mit Anti-D-Immunglobulinen}

Als erstes intravenöses Anti-D-Immunglobulin zur Behandlung der ITP wurde WinRho 1995 in den USA zugelassen. Die in den USA empfohlene Dosis für WinRho beträgt 50-75 $\mu \mathrm{g} / \mathrm{kg}$ i.v. für 1-3 Tage [4]. Zurzeit hat WinRho in der Bundesrepublik und in den anderen europäischen Ländern keine Zulassung. In der Hand erfahrener Therapeuten können WinRho und andere Anti-D-Präparate im Einzelfall als Therapieoption genutzt werden. Es wird berichtet, dass die subkutane (s.c.) Applikation von Anti-D-Immunglobulinen besser verträglich sei als die i.v. Gabe [66-68].

\section{Notfalltherapie mit Thrombozytenkonzentraten}

Die Gabe von Thrombozytenkonzentraten ist bei der Behandlung der ITP in der Regel nicht notwendig. Bei schweren Blutungen kann mit Thrombozytenkonzentraten jedoch ein kurzfristiger Anstieg der Thrombozytenzahl und ein Sistieren der Blutungen erreicht werden [69]. Die Recovery bzw. Verweildauer in der Zirkulation ist länger, wenn gleichzeitig hochdosiert Steroide und Immunglobuline verabreicht werden. Eine Stimulation der Bildung von Thrombozyten-Antikörpern wurde bisher nicht beobachtet. Die Thrombozytentransfusion ersetzt nicht eine spezifische Therapie der ITP (z.B. hoch dosierte Steroide und i.v. Immunglobuline).

\section{Begleitmaßnahmen}

- Medikamente: Thrombozytenfunktionshemmer (nichtsteroidale Antiphlogistika, Acetylsalicylsäure, Thienopyridine) sollten bei thrombozytopenen Patienten bis auf Einzelfälle (kürzliche Stentanlage, Schlaganfall, Angina pectoris, schwere periphere arterielle Verschlusskrankheit (pAVK)) vermieden werden.

- Eisenmangel und chronische Blutungsanämie: Manche ITPPatienten entwickeln durch chronische Blutungen, insbesondere verstärkte Menstruationsblutungen, einen Eisen- mangel und einen Abfall des Hämatokritwertes. Ein niedriger Hämatokrit verstärkt wiederum die Blutungsneigung. Daher sollten die Ferritinwerte regelmäßig überwacht werden. Vor allem bei Frauen im gebärfähigen Alter ist häufig eine orale Eisensubstitution indiziert, durch Hormonpräparate oder Antifibrinolytika (s.u.) können Menorrhagien reduziert werden.

- ITP-Patienten, die nicht schnell und dauerhaft auf die $1^{\text {st }}$ Line-Therapie ansprechen, sollten prophylaktisch geimpft werden (Pneumo-, Meningokokken, Haemophilus influenzae Typ b, Hepatitis B), weil die Notwendigkeit einer Splenektomie oder von Thrombozyten- und Bluttransfusionen und die damit verbundenen Infektionsrisiken nicht absehbar sind (s.u.).

\section{$2^{\text {nd }}$-Line-Therapie der persistierenden oder chronischen ITP}

Eine $2^{\text {nd }}$-Line-Therapie ist indiziert, wenn der Patient auf die $1^{\text {st }}$-Line-Therapie mit Steroiden nur partiell oder gar nicht anspricht und weiter blutet oder wenn er nach initialer Therapieansprache einen erneuten Thrombozytenabfall mit Blutungen entwickelt.

Die Indikation zur $2^{\text {nd }}$-Line-Therapie orientiert sich allein an der Blutungsneigung und nicht an der Thrombozytenzahl.

Bei allen Patienten mit persistierender oder chronischer ITP und WHO Grad-III- oder Grad-IV-Blutungen (Tab. 3) besteht eine Therapieindikation. Bei chronischen ITP-Patienten ohne (Grad 0) oder mit Grad-I/II-Blutungen besteht unabhängig von der Schwere der Thrombozytopenie keine zwingende Therapieindikation. Hier kann auch der Verlauf abgewartet werden.

\section{Erneute Therapie mit Steroiden}

Die Gabe von Steroiden ist zur Vorbereitung von operativen Eingriffen und zur Therapie akuter Blutungsepisoden (dann meist kombiniert mit i.v. Immunglobulinen) indiziert. Eine langfristige Steroidbehandlung sollte unbedingt vermieden werden.

\section{$2^{\text {nd }}$-Line-Therapie mit i.v. Immunglobulinen}

Die Gabe von i.v. Immunglobulinen ist im Rahmen der $2^{\text {nd }}-$ Line-Therapie zur Behandlung akuter Blutungsepisoden indiziert, wenn ein schneller Thrombozytenanstieg erreicht werden soll.

\section{H. pylori-Eradikation als $2^{\text {nd }}$-Line-Therapie}

Siehe dazu Abschnitt «Helicobacter pylori - Diagnostik und Therapie». 


\section{Splenektomie}

Die Splenektomie ist die $2^{\text {nd }}$-Line-Therapie mit der höchsten Rate an dauerhaften kompletten Remissionen.

Die Mitglieder des Expertenpanels sehen eine klare Indikation zur Splenektomie für alle Patienten mit persistierender oder chronischer Thrombozytopenie und Blutungen WHO Grad III, IV.

Die Mitglieder des Expertenpanels sehen keine zwingende Indikation zur Splenektomie für Patienten mit chronischer ITP, deren Thrombozytenzahlen $<30$ 000/ $\mu \mathrm{l}$ liegen und die keine oder nur leichte Blutungen (WHO Grad 0, I, II) haben. Hier muss individuell entschieden werden.

Es wird empfohlen, vor der Splenektomie alle bisher nicht erfolgten, notwendigen Impfungen (s.u.) durchzuführen und außerdem mit einer Knochenmarkspunktion die Diagnose zusätzlich abzusichern.

Die American Society of Hematology (ASH) Practice Guideline von 1996 hatte für alle Patienten mit persistierender Thrombozytopenie unter 20 000-30 000/ $\mu$ l eine zwingende Therapieindikation festgeschrieben, bis hin zur Splenektomie. Diese Empfehlung bestand unabhängig von der Blutungsneigung [1]. Die Mitglieder des Expertenpanels sehen es als nicht sinnvoll an, allein aufgrund niedriger Thrombozytenzahlen und unabhängig von der klinischen Blutungsneigung zur Splenektomie zu raten. Viele ITP-Patienten haben trotz niedriger Werte jahrelang keine oder nur minimale Blutungen. Hier muss die Indikation von individuellen Faktoren (bisherige Blutungsanamnese, Lebensstil, berufliches Blutungsrisiko, usw.) abhängig gemacht werden.

Morbidität und Mortalität der Splenektomie sind heute sehr niedrig. Es kommt jedoch immer noch zu Todesfällen ( $1 \%$ Mortalität bei Laparotomie, $0,1 \%$ bei Laparoskopie [4]; in anderen Publikationen bis 4\% [70]). Nach Splenektomie haben ITP-Patienten lebenslang ein erhöhtes Infektionsrisiko. Außerdem sind sie durch die vorhergehende Therapie mit Steroiden und anderen Immunsuppressiva zusätzlich gefährdet. Weitere seltene Nebenwirkungen werden berichtet:

- schwere, septische Infektionen (OPSI-Syndrom = overwhelming post-splenectomy infection) [71, 72],

- Thromboembolien [73-75],

- pulmonale Hypertonie [73, 76-78].

Es gibt viele Patienten, denen bisher ohne klinische Blutungsneigung, allein aufgrund der niedrigen Thrombozytenzahl eine Splenektomie angeraten wurde. Dies ist nicht zwingend notwendig, andererseits aber auch nicht kontraindiziert, wenn die Lebensumstände des Patienten und eine individuelle Abwägung des potenziellen Blutungsrisikos dies rechtfertigen.
Bei jungen Frauen mit möglichem Kinderwunsch muss berücksichtigt werden, dass durch die Splenektomie die Thrombozyten zwar ansteigen, dass die Bildung von Thrombozyten-Antikörpern aber in der Regel nicht beseitigt wird. Diese Antikörper können weiter diaplazentar übertragen werden und beim Fötus eine neonatale Thrombozytopenie auslösen [79, 80]. Die Thrombozytenzahl der splenektomierten Mutter korreliert nicht mit der Thrombozytenzahl des Kindes. Der betreuende Gynäkologe sollte bei splenektomierten ITP-Patientinnen trotz normaler oder nur leicht erniedrigter mütterlicher Thrombozytenwerte immer auf eine neonatale Thrombozytopenie vorbereitet sein (zur Behandlung von ITP-Patientinnen in der Schwangerschaft siehe Ref. [2, 4, 11, 12]).

Das größte Problem der Splenektomie ist jedoch, dass ein Drittel der Patienten kurzfristig oder auch nach Jahren einen therapiewürdigen Rückfall erleidet [42, 55, 81, 82].

Es ist bisher nicht möglich vorherzusagen, welcher Patient auf eine Splenektomie ansprechen wird und welcher nicht. Aufgrund widersprüchlicher publizierter Daten kann bisher auch nicht entschieden werden, ob der nuklearmedizinische Nachweis einer vermehrten Sequestration von markierten Thrombozyten in der Milz zur Vorhersage geeignet ist [8387]. Die neue internationale Konsensusleitlinie empfiehlt zwar die Splenektomie, wenn der Abbau markierter Thrombozyten vorwiegend in der Milz erfolgt [4]. Diese Empfehlung wird jedoch aufgrund der widersprüchlichen Daten (s.o.) vom Expertenpanel zurückhaltend beurteilt und nicht als verbindlich betrachtet. In der Bundesrepublik ist die nuklearmedizinische Untersuchung der Thrombozytensequestration nur an wenigen Zentren verfügbar.

Alle Patienten sollten - wenn es die Zeit zulässt - 4 Wochen vor der Operation oder 2 Wochen danach, wenn die Impfung vorher nicht möglich war, gegen Pneumokokken, Haemophilus influenzae Typ b und Meningokokken geimpft werden [4, 88]. Eine Hepatitis-B-Impfung empfiehlt sich ebenfalls bei allen ITP-Patienten, weil eine Bluttransfusion oder die Gabe von anderen Blutprodukten und das damit verbundene Infektionsrisiko für die Zukunft nie ausgeschlossen werden können.

Wenn nach der Splenektomie die Thrombozyten ansteigen, benötigen auch ITP-Patienten, solange sie immobilisiert sind, eine adäquate Thromboseprophylaxe mit niedermolekularem Heparin (s. aktuelle Thromboseprophylaxe-Leitlinie: www.awmf-leitlinien.de). Wenn die Thrombozytenzahl über 500 000/ $\mu \mathrm{l}$ ansteigt, kann außerdem Acetylsalicylsäure verabreicht werden.

\section{Zeitpunkt der Splenektomie}

Da auch bei Erwachsenen Spontanremissionen der ITP noch bis 12 Monate nach Diagnosestellung vorkommen können [7, 8] - danach sind sie außerordentlich selten [9] -, sollte versucht werden, die Splenektomie bis zu diesem Zeitpunkt hinauszuschieben. Außer bei therapierefraktären 
Patienten mit bedrohlichen (Grad III/IV) Blutungen ist dies in den allermeisten Fällen gut möglich. Gegebenenfalls kann eine sogenannte «Splenektomie aufschiebende» medikamentöse Therapie angeboten werden. Nach einem Jahr und bei persistierender Blutungsneigung sollte dann die Indikation zur Splenektomie erneut mit dem Patienten diskutiert werden.

\section{Medikamentöse Therapien in der $2^{\text {nd }}$-Line als Alternative zur} Splenektomie oder um eine Splenektomie aufzuschieben Azathioprin und Vincristin (s.u.) sind seit den 1960er- bzw. 1970er-Jahren verfügbar und haben eine sogenannte «Altzulassung» zur Behandlung der ITP. Eine Therapie mit diesen Substanzen als Alternative zur Splenektomie wäre somit grundsätzlich möglich. Der Anteil dauerhafter Remissionen ist jedoch nicht sehr hoch und die Nebenwirkungen von Azathioprin und Vincristin sind nicht unerheblich [89]. Dagegen lassen die Studiendaten für die neuen TRAs Romiplostim und Eltrombopag und auch für den Anti-CD20-Antikörper Rituximab eine deutlich bessere Wirksamkeit und geringere Toxizität erwarten.

Romiplostim und Eltrombopag sind in der Bundesrepublik für die Behandlung erwachsener, splenektomierter Patienten mit chronischer ITP zugelassen, die gegenüber anderen Therapien refraktär sind (z.B. Kortikosteroide, Immunglobuline). Die Anwendung von Romiplostim oder Eltrombopag kann als $2^{\text {nd }}$-Line-Therapie für erwachsene, nicht splenektomierte Patienten nur dann in Betracht gezogen werden, wenn eine Operation kontraindiziert ist (z.B. schlechter Allgemeinzustand, schwere Nebenerkrankungen, aktive Infektionen, Verwachsungen nach Voroperationen, Splenomegalie (spricht gegen Diagnose ITP), Adipositas permagna; s.a. [90]). Auch Rituximab wurde bisher häufig als «Splenektomie aufschiebende» Therapie eingesetzt [91-93], ohne dafür zugelassen zu sein. Es ist die Meinung der Panelmitglieder, dass bei klinischer Blutungsneigung eine medikamentöse $2^{\text {nd }}$-Line-Therapie mit Romiplostim, Eltrombopag oder Rituximab mit dem Patienten besprochen werden sollte, auch mit dem Ziel, eine Splenektomie aufzuschieben (s.o.). Dabei ist zu berücksichtigen, dass für Romiplostim und Eltrombopag randomisierte Phase-III-Studiendaten vorliegen [94, 95], während dies für eine $2^{\text {nd }}$-Line-Therapie mit Rituximab bisher nicht der Fall ist. Bei der Abwägung Rituximab oder TRAs der Splenektomie vorzuziehen, sollten die Patienten darauf hingewiesen werden, dass sich die Erfahrung mit diesen Therapien im Vergleich zur Splenektomie auf kleinere Patientenzahlen und einen noch relativ kurzen Beobachtungszeitraum beschränken, so dass möglicherweise bisher nicht alle Nebenwirkungen bekannt sind.

Die Patienten sind auf den fehlenden Zulassungsstatus beider Behandlungen vor Splenektomie hinzuweisen und über die Nebenwirkungsrisiken ausführlich aufzuklären.

\section{$3^{\text {rd }}$-Line-Therapie nach Splenektomie}

Ein Drittel der Patienten erleidet nach Splenektomie einen therapiewürdigen Rückfall [42, 55, 81, 82]. Dann sollte eine akzessorische Milz ausgeschlossen werden.

Bisher wurden in der $3^{\text {rd }}$-Line unterschiedliche immunsuppressive Therapien angeboten: Azathioprin, Cyclosporin, Mycophenolat, Vincristin usw. (Übersicht bei [96], Tab. 8).

Diese Situation hat sich mit der Entwicklung der TRAs Romiplostim (zugelassen seit Februar 2009) und Eltrombopag (zugelassen seit März 2010) geändert.

\section{Thrombopoietinrezeptor-Agonisten}

\section{Insuffiziente Thrombozytopoese und relativer}

\section{Thrombopoietinmangel bei ITP}

Bereits Anfang des letzten Jahrhunderts hatten deutsche Forscher die Meinung vertreten, dass bei einer ITP die Thrombozytenneubildung vermindert ist; die Idee wurde jedoch nicht weiter verfolgt $[97,98]$. In den Folgejahren dominierte dann die Vorstellung, dass bei der ITP der Thrombozytenabbau verstärkt und die Neubildung kompensatorisch erhöht ist [99]. Neuere Untersuchungen zeigen, dass bei fast der Hälfte der ITP-Patienten die Thrombozytopoese insuffizient ist [27, 28]. Es gibt Hinweise, dass sich Autoantikörper nicht nur gegen die Thrombozyten im zirkulierenden Blut, sondern auch gegen die Megakaryozyten im Knochenmark richten [30-33, 100]. Ein weiterer Befund ist der meist nur wenig erhöhte Thrombopoietinspiegel bei ITP-Patienten. Bei anderen Erkrankungen, die mit einem vergleichbaren Thrombozytenmangel einhergehen (z.B. bei aplastischer Anämie oder nach Chemotherapie), findet man deutlich höhere Spiegel. Man spricht deshalb von einem relativen Thrombopoietinmangel $[34,35]$.

\section{Romiplostim und Eltrombopag - Wirkung}

Die Erkenntnis, dass viele ITP-Patienten zusätzlich zum vermehrten Thrombozytenabbau eine insuffiziente Thrombozytopoese und einen relativen Thrombopoietinmangel haben, führte zum Einsatz von TRAs bei ITP [101]. 2008 wurden in den USA die Wirkstoffe Romiplostim (früher AMG531, Handelsname Nplate ${ }^{\circledR}$, Amgen GmbH, München, Deutschland) und Eltrombopag (früher SB-4971145-GR, Handelsname in den USA Promacta ${ }^{\mathrm{TM}}$, Handelsname in Europa Revolade ${ }^{\circledR}$, GlaxoSmithKline Gmbh und Co. KG, München, Deutschland) zugelassen. Romiplostim ist seit Februar 2009 in der Bundesrepublik zugelassen, Eltrombopag seit März 2010. 
Nach Meinung des Expertenpanels sollte sich die Entscheidung zur Therapie mit TRAs primär an der Blutungsneigung, nicht an der Thrombozytenzahl orientieren.

Bei splenektomierten und weiterhin thrombozytopenen Patienten mit WHO Grad-III- oder Grad-IV-Blutungen (Tab. 3) besteht eine Indikation für die Therapie mit TRAs.

Die Indikation zur Therapie mit TRAs bei splenektomierten, thrombozytopenen Patienten mit geringer oder gänzlich fehlender Blutungsneigung (WHO Blutungsgrade 0 , I und II) ist nicht gesichert. Hier sollen Nutzen und Risiken mit dem Patienten besprochen und eine individuelle Entscheidung gefunden werden.

Bei Thrombozytenzahlen $>50000 / \mu$ l besteht keine Indikation zur Therapie mit TRAs.

Romiplostim wird als s.c. Injektion einmal pro Woche verabreicht. Die Dosis wird der Thrombozytenzahl angepasst (Näheres s. Fachinformation). Es ist die Erfahrung der Panelmitglieder, dass zu Beginn einer Therapie die Thrombozytenwerte stark schwanken können. Viele Patienten erreichen jedoch im weiteren Verlauf eine relativ stabile Dosierung.

Eltrombopag wird oral verabreicht. Der Hersteller empfiehlt $4 \mathrm{~h}$ nach einer kalzium-, magnesium-, aluminium- oder eisenhaltigen Mahlzeit (Antazida, Milchprodukte, Vitamintabletten, Eisensubstitution bei Anämie) zu warten. Die Einnahme auf nüchternen Magen oder zur Nacht, vor dem $\mathrm{Zu}$ bettgehen, erscheint für viele Patienten am besten praktikabel. Auch bei Eltrombopag muss die Dosierung der Thrombozytenzahl angepasst werden.

Die TRAs Romiplostim und Eltrombopag erreichen bei ca. 80\% bisher therapierefraktärer Patienten einen Anstieg der Thrombozytenzahl über 50 000/ $\mu$ l. Ernsthafte Blutungen traten mit TRAs wesentlich seltener auf [93, 102-107]. Genauso wichtig ist, dass die meisten Patienten ihre bisherige immunsuppressive Medikation, zumeist Steroide, reduzieren oder ganz absetzen können. Dies bedeutet eine Reduzierung langfristiger Nebenwirkungen und einen erheblichen Gewinn an Lebensqualität [108-112]. Allerdings sind die Beobachtungszeiträume mit TRAs bisher noch relativ kurz (im Median unter einem Jahr) [44, 104, 107], und es ist unklar, ob TRAs unerwünschte Wirkungen induzieren können, die sich erst nach einer längeren Behandlungs- und Latenzzeit klinisch manifestieren.

Romiplostim und Eltrombopag - Nebenwirkungen und Risiken Die häufigsten Nebenwirkungen sind Kopf-, Gelenk- und Muskelschmerzen [113]. Wichtige, aber seltene Nebenwirkungen sind:

- Beim abrupten Absetzen von Romiplostim kann es zu einem überschießenden Abfall der Thrombozytenzahl unter die Ausgangswerte kommen. Dies wird bei Eltrombopag bisher nicht als medikamentenspezifischer Effekt beschrieben [114].

- Bei Eltrombopag wurden Leberwerterhöhungen berichtet [115].
- Sowohl unter der Behandlung mit Romiplostim als auch mit Eltrombopag wurden Thrombosen beobachtet [95, 104, 107, 116, 117]. Retrospektive Studien haben jedoch gezeigt, dass ITP-Patienten auch ohne TRAs ein erhöhtes Thromboserisiko tragen, insbesondere nach Splenektomie [43, 75, 118]. Insofern muss offen bleiben, ob TRAs das Risiko von Thrombosen und Embolien wirklich erhöhen. In Risikosituationen (z.B. bei Immobilisation, postoperativ) sollte eine adäquate Thromboseprophylaxe angeboten werden.

- Es liegen Einzelbefunde vor, die bei einigen wenigen Patienten eine leichtgradige Retikulinfaservermehrung im Knochenmark zeigen [116, 119, 120]. Aufgrund der bisher nur sehr begrenzten Daten ist es nicht möglich abzuschätzen, ob die Dauertherapie mit Thrombozyten-Wachstumsfaktoren eine Knochenmarksfibrose auslösen kann.

- Bei 2 Patienten mit Romiplostim wurden bisher neutralisierende Anti-Romiplostim-Antikörper beobachtet, die nach dem Absetzen von Romiplostim wieder verschwanden [104, 121]. Für Eltrombopag ist dergleichen bisher nicht beschrieben [107].

Folgende Untersuchungen sollten aufgrund der oben genannten Nebenwirkungsrisiken vor bzw. während der Behandlung mit TRAs durchgeführt werden:

- Vor Gabe von TRAs Untersuchung des Blutausstriches durch einen in der Diagnostik von hereditären Thrombozytopenien erfahrenen Arzt, zum sicheren Ausschluss alternativer Thrombozytopenieursachen (z.B. hereditäre Thrombozytopenien).

- Während der Therapie regelmäßige Kontrolle des Blutausstriches auf Veränderungen, die auf eine beginnende Knochenmarksfibrose hinweisen könnten (Normoblasten, «Tear-Drop»-Zellen).

- Aufgrund der bisher noch begrenzten Datenlage empfiehlt das Expertengremium eine Knochenmarkspunktion mit zusätzlicher Retikulinfaserfärbung vor Beginn einer Therapie mit Thrombopoietinanaloga und eine erneute Punktion nach 1 Jahr.

- Patienten mit Thrombophilie oder Thrombosen in der Anamnese waren von den Zulassungsstudien ausgeschlossen, und das Expertengremium empfiehlt, in diesem Fall die Indikation zur Gabe von TRAs sehr kritisch zu stellen.

- Bei der Behandlung mit Eltrombopag sollten die Leberwerte regelmäßig kontrolliert werden.

- Patienten asiatischer Herkunft sprechen stärker auf Eltrombopag an und sollten initial mit einer niedrigeren Dosis behandelt werden (25 mg, Weiteres s. Fachinfo) [122].

\section{Rituximab}

Rituximab ist in keinem Land der Welt zur Behandlung der ITP zugelassen, dennoch wird es in Fachkreisen als eine etablierte Therapie bei chronischer ITP betrachtet. Auch in der Bundesrepublik werden zahlreiche ITP-Patienten trotz fehlender Zulassung mit Rituximab behandelt. Deshalb soll im Folgenden auf diese Therapie eingegangen werden. 
Rituximab erreicht bei ca. 30-50\% der chronischen ITPPatienten langfristige - definiert als über 12 Monate anhaltende - Remissionen [93, 123-126].

Die übliche Dosis liegt bei $375 \mathrm{mg} / \mathrm{m}^{2} 1 \times$ pro Woche für 4 aufeinanderfolgende Wochen. Es ist bisher nicht geklärt, ob niedrigere Dosen gleich wirksam sind [127-129].

Die Behandlung mit Rituximab ist in der Regel gut verträglich. Es wurden aber auch ernste Nebenwirkungen berichtet, insbesondere Infektionen [123, 130, 131]. Es muss offen bleiben, inwieweit diese auf Rituximab oder die immunsuppressive Vor- und Begleittherapie zurückzuführen waren.

\section{Weitere $3^{\text {rd }}$-Line-Therapien (alphabetisch)}

Die weiteren Therapieoptionen kommen in der Regel nur dann in Frage, wenn Splenektomie, TRAs und eventuell auch Rituximab kein ausreichendes Ansprechen der Blutungsneigung erreichen. Bei der Auswahl der Therapie besteht keine Präferenz, die Entscheidung muss individuell getroffen werden.

\section{Azathioprin}

Die Dosis beträgt $2 \mathrm{mg} / \mathrm{kg} / \mathrm{Tag}$. Neutropenien kommen vor, die Leukozytenzahl muss zu Beginn der Therapie regelmäßig (z.B. alle 2-4 Wochen) kontrolliert werden. Diese Therapie sollte mindestens 4 Monate lang probiert werden, bis von einer Nichtansprache ausgegangen werden kann [132, 133].

\section{Cyclophosphamid}

Sowohl die orale Dauertherapie (1-2 mg/kg/Tag) als auch die i.v. Behandlung $\left(0,3-1 \mathrm{~g} / \mathrm{m}^{2}, 1-3\right.$ Dosen alle $2-4$ Wochen) werden beschrieben. Neben den üblichen Zytostatikanebenwirkungen (Knochenmarksuppression usw.) muss der Patient auch über das Risiko von Blasenkrebs und sekundären Leukämien aufgeklärt werden. Die Fertilität kann eingeschränkt werden. Cyclophosphamid hat in der Bundesrepublik keine arzneimittelrechtliche Zulassung für die Therapie der ITP.

\section{Cyclosporin A}

Cyclosporin A (2,5-3 mg/kg/Tag) wird als Monotherapie oder in Kombination mit Prednison eingesetzt. Die Therapienebenwirkungen können gerade bei älteren Patienten belasten. In Studien werden Zielspiegel von 100-400 ng/ml angestrebt $[134,135]$. Cyclosporin A hat in der Bundesrepublik keine arzneimittelrechtliche Zulassung für die Therapie der ITP.

\section{Danazol}

Die Dosis beträgt 400-800 mg/Tag. Eine Therapieansprache ist in 4-6 Wochen zu erwarten, danach sollte versucht werden, die Dosis zu reduzieren. Danazol ist ein modifiziertes Androgen und bei längerfristiger Therapie muss auf die Leberfunktion geachtet werden. Weitere Nebenwirkungen sind Gewichtszunahme, Myalgien, Haarverlust [136]. Danazol hat in der Bundesrepublik keine arzneimittelrechtliche Zulassung und wird auch nicht vertrieben. Es muss über die Auslandsapotheke importiert werden (Danol, Kapseln à 200 mg).

\section{Dapson}

Die Dosis beträgt 75-100 mg/Tag. Diese Therapie spricht manchmal nur sehr langsam an und sollte deshalb mindestens 2 Monate lang versucht werden. Nach Splenektomie sind die Ansprechraten noch geringer [137-139]. Bei Patienten aus mediterranen Ländern und besonders bei Farbigen muss ein Mangel oder Defekt der Glukose-6-PhosphatDehydrogenase vorher ausgeschlossen werden. Dapson (DAPSON-Fatol, Tabletten à $50 \mathrm{mg}$ ) hat in der Bundesrepublik keine arzneimittelrechtliche Zulassung für die Therapie der ITP.

\section{Mycophenolat mofetil}

Man gibt zuerst $250 \mathrm{mg} 2 \times$ täglich (tgl.), nach 2 Wochen wird die Dosis auf $2 \times 500 \mathrm{mg} /$ Tag erhöht, nach 4 Wochen auf $2 \times$ 1000 mg/Tag, eine eventuelle Steroidtherapie kann gleichzeitig reduziert werden. Mycophenolat hat in der Bundesrepublik keine arzneimittelrechtliche Zulassung für die Therapie der ITP [140-142].

\section{Vincristin/Vinblastin}

Vincristin wird mit einer Dosis von 1-2 mg $1 \times$ pro Woche für 4-6 Wochen verabreicht, Vinblastin mit 5-10 mg. Häufige Nebenwirkungen sind die zytostatikainduzierte Thrombozytopenie, Neuropathie, Obstipation. Die Wirkung hält in der Regel nicht lange an [143-146].

Weitere experimentelle Therapieverfahren oder Fallberichte - Alemtuzumab/Campath 1H [147, 148],

- Kombinationschemotherapie (CVP) [149-151] oder Kombination mehrerer Immunsuppressiva [152],

- Stammzelltransplantation [153-156].

\section{Unwirksame oder nicht indizierte Therapien}

Folgende Therapien sind unwirksam und sollten nicht mehr eingesetzt werden [4]:

- Colchicin,

- Interferon $\alpha$,

- Protein-A-Immunoadsorption,

- alleinige Plasmapherese,

- Vitamin C.

\section{Antifibrinolytika}

Durch die Gabe der Fibrinolyseinhibitoren Tranexamsäure (Cyklokapron $^{\circledR}$, Filmtabletten à $500 \mathrm{mg}, 20-25 \mathrm{mg} / \mathrm{kg}$ alle $8 \mathrm{~h}$ per os (p.o.)) oder von $\varepsilon$-Aminocapronsäure (Amicar ${ }^{\circledR}$, über die Auslandsapotheke erhältlich als Tabletten à 500 mg; Dosierung: $50-60 \mathrm{mg} / \mathrm{kg}$ alle $4-6 \mathrm{~h}$ p.o.) kann bei leichten Schleimhautblutungen, bei Menorrhagien und im Rahmen von Zahneingriffen häufig eine ausreichende Blutstillung erreicht werden [157]. Bei Blutungen in das Auge, das Zentralnervensystem (ZNS) oder bei Organblutungen sind Antifibrinolytika nicht ausreichend wirksam. 
Tab. 9. Anzustrebende Thrombozytenwerte vor operativen Eingriffen
Zahnärztliche Zahnreinigung, Zahnsteinentfernung

Zahnextraktion (einfach)

Zahnextraktion (komplex, z.B. Molar)

Leitungsanästhesie bei Zahneingriff

Lumbalpunktion

Spinalanästhesie

Epiduralanästhesie

Gastrointestinale Endoskopie mit Biopsie ${ }^{\mathrm{a}}$

Bronchoskopie/Bronchiallavage

Bronchoskopie mit transbronchialer Biopsie

Andere Organpunktionen/Biopsien ${ }^{\mathrm{b}}$

Kleine Operation ${ }^{\mathrm{c}}$

Größere Operation ${ }^{c}$

Neurochirurgischer Eingriff

Eingriffe am hinteren Augenabschnitt
$>20000-30000 / \mu 1$

$>30000 / \mu 1$

$>50000 / \mu 1$

$>30000 / \mu 1$

$>50000 / \mu 1$

$>50000 / \mu \mathrm{l}$

$>80000 / \mu 1$

$>20000 / \mu 1$

$>20000 / \mu 1$

$>50000 / \mu 1$

$>50000 / \mu 1$

$>50000 / \mu 1$

$>80000 / \mu 1$

$70000-100000 / \mu 1$

$70000-100000 / \mu 1$

${ }^{a}$ Gastrointestinale Endoskopie ohne Biopsie auch bei sehr niedrigen Werten möglich.

${ }^{\mathrm{b}}$ Knochenmarksbiopsie auch bei sehr niedrigen Werten möglich.

${ }^{\mathrm{c}}$ Größere Operationen sind z.B. abdominelle oder thoraxchirurgische Eingriffe und Operationen in Regionen, die im Falle einer postoperativen Blutung nicht komprimiert werden können. Anmerkung: Diese Zahlen wurden für Patienten mit Bildungsstörung erhoben. Für ITP-Patienten gibt es keine entsprechenden Daten. Hier muss auch die individuelle Blutungsanamnese berücksichtigt werden, ob ein Patient auch schon bei höheren Thrombozytenzahlen geblutet hat (s.a. Abschnitt «Vorgehen bei Operationen und Zahneingriffen»).

Tab. 10. Selbsthilfegruppen in der Bundesrepublik und international

\begin{tabular}{lll}
\hline Gruppe & Webseite & Kontaktperson \\
\hline ITP-SHG Berlin & & Fr. Plonka (n.plonka@gmx.de) \\
ITP-SHG Gießen & www.itp-information.de & Fr. Arnold (über Homepage) \\
ITP-SHG Hamburg & www.morbus-werlhof.de & Fr. Hass (über Homepage) \\
ITP-SHG Sömmerda & Fr. Riese (s-riese@t-online.de) \\
USA: Platelet Disorder Support Organisation & www.pdsa.org & \\
Großbritannien: ITP Support Association & www.itpsupport.org.uk & \\
\hline
\end{tabular}

\section{Vorgehen bei Operationen und Zahneingriffen}

Wenn bei Patienten mit ITP eine Operation oder ein invasiver diagnostischer Eingriff elektiv geplant werden kann, dann sollten durch eine vorbereitende Therapie die in Tabelle 9 dargestellten Thrombozytenwerte erreicht werden [158-161].

\section{Impfungen}

Akute ITP-Fälle in Assoziation mit Impfungen wurden beschrieben. Fälle von chronischer ITP sind jedoch so selten, dass es sich auch um zufällige Assoziationen handeln könnte $[162,163]$. Es muss deshalb offen bleiben, ob bei Patienten, die früher eine ITP hatten und die jetzt in Remission sind, oder bei Patienten, die aktuell eine chronische ITP haben, eine Impfung einen Rückfall oder eine Verschlimmerung der Erkrankung hervorrufen kann. Wenn keine Impfungen erfolgen und die Patienten dann an einer Virusinfektion erkranken, besteht ebenfalls und möglicherweise sogar ein höheres Risiko, dass diese Infektion eine Verminderung der Throm- bozytenzahl herbeiführt [164]. Nur wenn die chronische ITP ursprünglich nach einer Impfung auftrat - solche Fälle wurden beschrieben - dann sollten weitere Impfungen mit diesem oder anderen Impfstoffen, die ähnliche Bestandteile enthalten, vermieden werden. Lebendimpfungen sind bei immunsupprimierten Patienten in der Regel kontraindiziert.

\section{Patientenselbsthilfe}

Informationen und Kontaktadressen zur Patientenselbsthilfe können Tabelle 10 entnommen werden.

\section{Abschließende Stellungnahme}

Die Autoren haben versucht, in diesem Manuskript den aktuellen Kenntnisstand wiederzugeben. Sämtliche Aussagen sind als Empfehlungen zu verstehen und rechtlich nicht bindend. Sie haben daher weder haftungsbegründende noch haftungsbefreiende Wirkung. 


\section{Literatur}

1 George JN, Woolf SH, Raskob GE, Wasser JS, Aledort LM, Ballem PJ, Blanchette VS, Bussel JB, Cines DB, Kelton JG, Lichtin AE, McMillan R, Okerbloom JA, Regan DH, Warrier I: Idiopathic thrombocytopenic purpura: a practice guideline developed by explicit methods for the American Society of Hematology. Blood 1996;88:3-40.

$\checkmark 2$ British Committee for Standards in Haematology General Haematology Task Force: Guidelines for the investigation and management of idiopathic thrombocytopenic purpura in adults, children and in pregnancy. Br J Haematol 2003;120:574-596.

3 Hiller E, Matzdorff A, Rummel M: Thrombozytopenien; in Dierkesmann R, Fleig WE, Heidrich $\mathrm{H}$, Heimpel Hkern WV, Meyer J, Müller OA, Specker CH, Wanner C (Hrsg): Rationelle Diagnostik und Therapie in der Inneren Medizin. Leitlinien-basierte Empfehlungen für die Praxis. München, Urban und Fischer Verlag, 2008, B3, S. 1-6.

4 Provan D, Stasi R, Newland AC, Blanchette VS Bolton-Maggs P, Bussel JB, Chong BH, Cines DB, Gernsheimer TB, Godeau B, Grainger J, Greer I, Hunt BJ, Imbach PA, Lyons G, McMillan R, Rodeghiero F, Sanz MA, Tarantino M, Watson S, Young J, Kuter DJ: International consensus report on the investigation and management of primary immune thrombocytopenia. Blood 2010;115:168-186.

$\checkmark 5$ Rodeghiero F, Stasi R, Gernsheimer T, Michel M, Provan D, Arnold DM, Bussel JB, Cines DB, Chong BH, Cooper N, Godeau B, Lechner K, Mazzucconi MG, McMillan R, Sanz MA, Imbach P, Blanchette V, Kühne T, Ruggeri M, George JN: Standardization of terminology, definitions and outcome criteria in immune thrombocytopenic purpura (ITP) of adults and children: Report from an international working group. Blood 2009;113: 2386-2393.

6 Cines DB, Bussel JB, Liebman HA, Prak ETL: The ITP syndrome: pathogenic and clinical diversity. Blood 2009;113:6511-6221.

7 Stasi R, Stipa E, Masi M, Cecconi M, Scimò MT, Oliva F, Sciarra A, Perrotti AP, Adomo G, Amadori S, Papa G: Long-term observation of 208 adults with chronic idiopathic thrombocytopenic purpura. Am J Med 1995;98:436-442.

$>8$ Sailer T, Lechner K, Panzer S, Kyrle PA, Pabinger I: The course of severe autoimmune thrombocytopenia in patients not undergoing splenectomy. Haematologica 2006;91:1041-1045.

-9 Simanek R, Panzer S, Lechner K, Pabinger I: Late spontaneous remissions in severe adult autoimmune thrombocytopenia. Ann Hematol 2007;86: 705-710.

10 Dickerhoff R, Gaedicke G, Sutor H: Leitlinie K4a: Akute Immun-Thrombozytopenie (ITP); in Deutsche Gesellschaft für Kinderheilkunde und Jugendmedizin (Hrsg): Leitlinien Kinderheilkunde und Jugendmedizin. München, Urban und Fischer Verlag, 2006.

11 McCrae KR, Samuels P, Schreiber AD: Pregnancy associated thrombocytopenia: Pathogenesis and management. Blood 1992;80:2697-2714.

12 Webert KE, Mittal R, Sigouin C, Heddle NM, Kelton JG: A retrospective 11-year analysis of obstetric patients with idiopathic thrombocytopenic purpura. Blood 2003;102:4306-4311.

13 Abrahamson PE, Hall SA, Feudjo-Tepie M, Mitrani-Gold FS, Logie J: The incidence of idiopathic thrombocytopenic purpura among adults: a population-based study and literature review. Eur J Haematol 2009;83:83-89.
4 Feudjo-Tepie MA, Robinson NJ, Bennett D: Prevalence estimates of adult chronic idiopathic thrombocytopenic purpura (ITP) in the United States. J Thromb Haemost 2008;6:711-712.

15 Frederiksen H, Schmidt K: The incidence of idiopathic thrombocytopenic purpura in adults increases with age. Blood 1999;94:909-913.

16 Neylon AJ, Saunders PW, Howard MR, Proctor SJ, Taylor PR; Northern Region Haematology Group: Clinically significant newly presenting autoimmune thrombocytopenic purpura in adults: a prospective study of a population-based cohort of 245 patients. Br J Haematol 2003;122:966-974

17 Segal JB, Powe NR: Prevalence of immune thrombocytopenia: analyses of administrative data. J Thromb Haemost 2006;4:2377-2383.

18 Schoonen WM, Kucera G, Coalson J, Li L, Rutstein M, Mowat F, Fryzek J, Kaye JA: Epidemiology of immune thrombocytopenic purpura in the General Practice Research Database. Br J Haematol 2009;145:235-244.

19 Meyer O, Agaylan A, Kiesewetter H, Salama A: A novel antigen-specific capture assay for the detection of platelet antibodies and HPA-1a phenotyping. Vox Sang 2006;91:324-330.

20 Olsson B, Andersson PO, Jernås M, Jacobsson S, Carlsson B, Carlsson LM, Wadenvik H: T-cellmediated cytotoxicity toward platelets in chronic idiopathic thrombocytopenic purpura. Nat Med 2003;9:1123-1124.

21 Olsson B, Ridell B, Carlsson L, Jacobsson S, Wadenvik H: Recruitment of $\mathrm{T}$ cells into bone marrow of ITP patients possibly due to elevated expression of VLA-4 and CX3CR1. Blood 2008; 112:1078-1084.

22 Stasi R, Cooper N, Del Poeta G, Stipa E, Laura Evangelista M, Abruzzese E, Amadori S: Analysis of regulatory T-cell changes in patients with idiopathic thrombocytopenic purpura receiving B celldepleting therapy with rituximab. Blood 2008; 112:1147-1150

23 Yu J, Heck S, Patel V, Levan J, Yu Y, Bussel JB, Yazdanbakhsh K: Defective circulating CD25 regulatory $\mathrm{T}$ cells in patients with chronic immune thrombocytopenic purpura. Blood 2008;112:13251328.

24 Cines DB, Blanchette VS: Immune thrombocytopenic purpura. N Engl J Med 2002;346:995-1008.

25 Zhang W, Nardi MA, Borkowsky W, Li Z, Karpatkin S: Role of molecular mimicry of hepatitis C virus protein with platelet GPIIIa in hepatitis Crelated immunologic thrombocytopenia. Blood 2009;113:4086-4093.

26 Ballem PJ, Segal GM, Stratton J, Gernsheimer T, Adamson JW, Slichter SJ: Mechanism of thrombocytopenia in chronic autoimmune thrombocytopenic purpura. Evidence of both impaired platelet production and increased platelet clearance. J Clin Invest 1987;80:33-40.

27 Louwes H, Zeinali Lathori OA, Vellenga E, de Wolf JT: Platelet kinetic studies in patients with idiopathic thrombocytopenic purpura. Am J Med 1999;106:430-434.

28 Siegel RS, Rae JL, Barth S, Coleman RE, Reba RC, Kurlander R, Rosse WF: Platelet survival and turnover: important factors in predicting response to splenectomy in immune thrombocytopenic purpura. Am J Hematol 1989;30:206-212.

29 Alimardani G, Guichard J, Fichelson S, Cramer EM: Pathogenic effects of anti-glycoprotein Ib antibodies on megakaryocytes and platelets. Thromb Haemost 2002;88:1039-1046.
30 Chang M, Nakagawa PA, Williams SA, Schwartz MR, Imfeld KL, Buzby JS, Nugent DJ: Immune thrombocytopenic purpura (ITP) plasma and purified ITP monoclonal autoantibodies inhibit megakaryocytopoiesis in vitro. Blood 2003;102:887-895.

31 Houwerzijl EJ, Blom NR, van der Want JJL, Vellenga E, de Wolf JT: Ultrastructural study shows morphologic features of apoptosis and para-apoptosis in megakaryocytes from patients with idiopathic thrombocytopenic purpura. Blood 2004;103: 500-506.

32 McMillan R, Luiken GA, Levy R, Yelenosky R, Longmire RL: Antibody against megakaryocytes in idiopathic thrombocytopenic purpura. JAMA 1978;239:2460-2462.

-33 McMillan R, Wang L, Tomer A, Nichol J, Pistillo J: Suppression of in vitro megakaryocyte production by antiplatelet autoantibodies from adult patients with chronic ITP. Blood 2004;103:1364-1369.

34 Emmons RV, Reid DM, Cohen RL, Meng G, Young NS, Dunbar CE, Shulman NR: Human thrombopoietin levels are high when thrombocytopenia is due to megakaryocyte deficiency and low when due to increased platelet destruction. Blood 1996;87:4068-4071.

35 Nichol JL: Endogenous TPO (eTPO) levels in health and disease: possible clues for therapeutic intervention. Stem Cells 1998;16(suppl 2):165-175.

36 Bolton-Maggs PHB, Moon I: National audit of the management of childhood idiopathic thrombocytopenic purpura against UK guidelines: closing the loop - education and re-audit demonstrate a change in practice. Blood 2001;98(suppl 1, part 2): 58 b, abstr 3847.

37 Buchanan GR, Adix L: Grading of hemorrhage in children with idiopathic thrombocytopenic purpura. J Pediatr 2002;141:683-688.

38 Khellaf M, Michel M, Schaeffer A, Bierling P, Godeau B: Assessment of a therapeutic strategy for adults with severe autoimmune thrombocytopenic purpura based on a bleeding score rather than platelet count. Haematologica 2005;90:829-832.

39 Page LK, Psaila B, Provan D, Michael Hamilton J, Jenkins JM, Elish AS, Lesser ML, Bussel JB: The immune thrombocytopenic purpura (ITP) bleeding score: assessment of bleeding in patients with ITP. Br J Haematol 2007;138:245-248.

40 Djulbegovic B, Cohen Y: The natural history of refractory idiopathic thrombocytopenic purpura. Blood 2001;98:2282-2283.

41 Cohen YC, Djulbegovic B, Shamai-Lubovitz O, Mozes B: The bleeding risk and natural history of idiopathic thrombocytopenic purpura in patients with persistent low platelet counts. Arch Intern Med 2000;160:1630-1638.

42 Portielje JE, Westendorp RG, Kluin-Nelemans HC, Brand A: Morbidity and mortality in adults with idiopathic thrombocytopenic purpura. Blood 2001;97:2549-2554.

43 Aledort LM, Hayward CP, Chen MG, Nichol JL, Bussel J; ITP Study Group: Prospective screening of 205 patients with ITP, including diagnosis, serological markers, and the relationship between platelet counts, endogenous thrombopoietin, and circulating antithrombopoietin antibodies. Am J Hematol 2004;76:205-213. 
44 Khellaf M, Quittet P, Viallard JF, Alexis M, Cheze S, Durang JM, Lefrere F, Galicier L, Lambotte O, Panelatti G, Rossi JF, Slama B, Damaj G, Sebahoun G, Gyan E, Delbrel X, Dhedin N, Royer B, Schleinitz N, Roudot-Thoraval F, Mahévas M, Languille L, Bierling P, Michel M, Godeau B: Safety and efficacy of romiplostim in immune thrombocytopenia (ITP) in the «real-life»: Result of the French experience in 72 adults. Blood 2009; 114:1365, abstr 3519

-45 Soupir CP, Vergilio JA, Kelly E, Dal Cin P, Kuter D, Hasserjian RP: Identification of $\operatorname{del}(20 q)$ in a subset of patients diagnosed with idiopathic thrombocytopenic purpura. Br J Haematol 2009;144:794805.

-46 Noris P, Klersy C, Zecca M, Arcaini L, Pecci A, Melazzini F, Terulla V, Bozzi V, Ambaglio C, Passamonti F, Locatelli F, Balduini CL: Platelet size distinguishes between inherited macrothrombocytopenias and immune thrombocytopenia. J Thromb Haemost 2009;7:2131-2136.

47 Arnold DM, Bernotas A, Nazi I, Stasi R, Kuwana M, Liu Y, Kelton JG, Crowther MA: Platelet count response to $H$. pylori treatment in patients with immune thrombocytopenic purpura with and without H. pylori infection: a systematic review. Haematologica 2009;94:850-856.

48 Emilia G, Luppi M, Zucchini P, Morselli M, Potenza L, Forghieri F, Volzone F, Jovic G, Leonardi G, Donelli A, Torelli G: Helicobacter pylori infection and chronic immune thrombocytopenic purpura: long-term results of bacterium eradication and association with bacterium virulence profiles. Blood 2007;110:3833-3841.

49 Kohda K, Kuga T, Kogawa K, Kanisawa Y, Koike K, Kuroiwa G, Hirayama Y, Sato Y, Niitsu Y: Effect of Helicobacter pylori eradication on platelet recovery in Japanese patients wtih chronic idiopathic thrombocytopenic purpura and secondary autoimmune thrombocytopenic purpura. Br J Haematol 2002;118:584-588.

50 Michel M, Cooper N, Jean C, Frissora C, Bussel JB: Does Helicobater pylori initiate or perpetuate immune thrombocytopenic purpura? Blood 2004; 103:890-896.

51 Stasi R, Provan D: Helicobacter pylori and chronic ITP. Hematol Am Soc Hematol Educ Program 2008:206-211.

52 Stasi R, Sarpatwari A, Segal JB, Osborn J, Evangelista ML, Cooper N, Provan D, Newland A, Amadori S, Bussel JB: Effects of eradication of Helicobacter pylori infection in patients with immune thrombocytopenic purpura: a systematic review. Blood 2009:113:1231-1240.

53 Berchtold P, Müller D, Beardsley D, Fujisawa K, Kaplan C, Kekomäki R, Lipp E, Morell-Kopp MC, Kiefel V, McMillan R, von dem Borne AE, Imbach $\mathrm{P}$ : International study to compare antigen-specific methods used for the measurement of antiplatelet autoantibodies. Br J Haematol 1997;96:477-483.

54 Cortelazzo S, Finazzi G, Buelli M, Molteni A, Viero P, Barbui T: High risk of severe bleeding in aged patients with chronic idiopathic thrombocytopenic purpura. Blood 1991;77:31-33.

55 Vianelli N, Valdrè L, Fiacchini M, de Vivo A, Gugliotta L, Catani L, Lemoli RM, Poli M, Tura S: Long-term follow-up of idiopathic thrombocytopenic purpura in 310 patients. Haematologica 2001; 86:504-509.
56 Bellucci S, Charpak V, Chastang C, Tobelem G: Low doses $\mathrm{v}$ conventional doses of corticoids in immune thrombocytopenic purpura (ITP): Results of a randomized clinical trial in 160 children, 223 adults. Blood 1988;71:1165-1169.

57 George JN, El-Harake MA, Raskob GE: Chronic idiopathic thrombocytopenic purpura. N Engl J Med 1994;331:1207-1211.

58 Pizzuto J, Ambriz R: Therapeutic experience on 934 adults with idiopathic thrombocytopenic purpura: Multicentric trial of the cooperative Latin American group on hemostasis and thrombosis. Blood 1984;64:1179-1183.

59 Bussel JB, Pham LC: Intravenous treatment with gammaglobulin in adults with immune thrombocytopenic purpura: review of the literature. Vox Sang 1987;52:206-210.

60 Godeau B, Caulier MT, Decuypere L, Bierling P: Intravenous immunoglobulin for adults with autoimmune thrombocytopenic purpura: results of a randomized trial comparing 0.5 and $1 \mathrm{~g} / \mathrm{kg}$ body weight. Br J Haematol 1999;107:716-719.

61 Godeau B, Chevret S, Varet B, Lefrère F, Zini JM, Bassompierre F, Chèze S, Legouffe E, Hulin C, Grange MJ, Fain O, Bierling P; French ATIP Study Group: Intravenous immunoglobulin or high-dose methylprednisolone, with or without oral prednisone, for adults with untreated severe autoimmune thrombocytopenic purpura: a randomised, multicentre trial. Lancet 2002;359:23-29.

62 Caress JB, Hobson-Webb L, Passmore LV, Finkbiner AP, Cartwright MS: Case-control study of thromboembolic events associated with IV immunoglobulin. J Neurol 2009;256:339-342.

63 Rault R, Piraino B, Johnston JR, Oral A: Pulmonary and renal toxicity of intravenous immunoglobulin. Clin Nephrol 1991;36:83-86.

64 Ruggeri M, Castaman G, De Nardi G, Rodeghiero F: Acute renal failure after high-dose intravenous immune globulin in a patient with idiopathic thrombocytopenic purpura. Haematologica 1993; 78:338-339.

65 Woodruff RK, Grigg AP, Firkin FC, Smith IL: Fatal thrombotic events during treatment of autoimmune thrombocytopenia with intravenous immunoglobulin in elderly patients. Lancet 1986;2: 217-218.

66 Kjaersgaard M, Edslev PW, Hasle H: Subcutaneous anti-D treatment of idiopathic thrombocytopenic purpura in children. Pediatr Blood Cancer 2009;53:1315-1317.

67 Meyer O, Kiesewetter H, Hermsen M, Salama A: Efficacy and safety of anti-D given by subcutaneous injection to patients with autoimmune thrombocytopenia. Eur J Haematol 2004;73:71-72.

68 Trebo M, Frey E, Gradner H, Minkov M: Subcutaneous anti-D globulin application is a safe treatment option of immune thrombocytopenia in children. Ann Hematol 2010;89:415-418.

69 Salama A, Kiesewetter H, Kalus U, Movassaghi K, Meyer O: Massive platelet transfusion is a rapidly effective emergency treatment in patients with refractory autoimmune thrombocytopenia. Thromb Haemost 2008;100:762-765.

70 Jaglowski SM, Byrd JC, Jones JA: In-hospital mortality and trends associated with splenectomy in patients with immune-mediated thrombocytopenia (ITP). Blood 2009;114:569, abstr 1398.

71 Lortan JE: Management of asplenic patients. Br J Haematol 1993;84:566-569.

72 Schilling RF: Estimating the risk for sepsis after splenectomy in hereditary spherocytosis. Ann Intern Med 1995;122:187-188.
73 Crary SE, Buchanan GR: Vascular complications after splenectomy for hematologic disorders. Blood 2009;114:2861-2868.

74 Schneemilch M, Mohren M, Fahlke J, Lippert H, Pross M: Thromboembolic complications after splenectomy: a rare but serious complication. Onkologie 2008;31(suppl 4):194, abstr V561.

75 Yong M, Thomsen RW, Schoonen WM, Farkas DK, Riis A, Fryzek JP, Sørensen HAT: Mortality risk in splenectomised patients: a Danish population-based cohort study. Eur J Intern Med 2010; 21:12-16.

76 Bonderman D, Jakowitsch J, Adlbrecht C, Schemper M, Kyrle PA, Schönauer V, Exner M, Klepetko W, Kneussl MP, Maurer G, Lang I: Medical conditions increasing the risk of chronic thromboembolic pulmonary hypertension. J Thromb Haemost 2005;93:512-516.

77 Hoeper MM, Niedermeyer J, Hoffmeyer F, Flemming P, Fabel H: Pulmonary hypertension after splenectomy? Ann Intern Med 1999;130:506-509.

78 Jaï X, Ioos V, Jardim C, Sitbon O, Parent F, Hamid A, Fadel E, Dartevelle P, Simonneau G, Humbert M: Splenectomy and chronic thromboembolic pulmonary hypertension. Thorax 2005;60: 1031-1034.

79 Piscitelli JT, Simel DL, Rosse WF: Does maternal platelet-associated or platelet-bindable IgG correlate with levels in umbilical cord blood or colostrum during normal pregnancy? Am J Obstet Gynecol 1988;158:430-434.

80 Yamada H, Fujimotos S: Perinatal management of idiopathic thrombocytopenic purpura in pregnancy: risk factors for passive immune thrombocytopenia. Ann Hematol 1994;68:39-42.

81 Bell WR: Long-term outcome of splenectomy for idiopathic thrombocytopenic purpura. Semin $\mathrm{He}$ matol 2000;37(suppl 1):22-25.

82 Kojouri K, Vesely SK, Terrell DR, George JN: Splenectomy for adult patients with idiopathic thrombocytopenic purpura: a systematic review to assess long-term platelet count responses, prediction of response, and surgical complications. Blood 2004;104:2623-2634.

83 Cugliotta L, Isacchi G, Guarini A, Ciccone F, Motta MR, Lattarini C, Bachetti G, Mazzucconi MG, Baccarani M, Mandelli F, Tura S: Chronic idiopathic thrombocytopenic purpura (ITP): site of platelet sequestration and results of splenectomy. A study of 197 patients. Scand J Haematol 1981;

26:407-412.
84 Juliá A, Araguás C, Rosselló J, Bueno J, Domenech P, Olona M, Guardia R, Petit J, Flores A Lack of useful clinical predictors of response to splenectomy in patients with chronic idiopathic thrombocytopenic purpura. Br J Haematol 1990; 76:250-255.

85 Najean Y, Rain JD, Billotey C: The site of destruction of autologous ${ }^{111}$ In-labelled platelets and the efficiency of splenectomy in children and adults with idiopathic thrombocytopenic purpura: a study of 578 patients with 268 splenectomies. Br J Haematol 1997;97:547-550.

86 Rossi G, Cattaneo C, Motta M, Pizzocaro C, Lanzi $\mathrm{S}$, Pouchè A: Platelet kinetic study in patients with idiopathic thrombocytopenic purpura (ITP) refractory or relapsing after corticosteroid treatment. Hematol J 2002;3:148-152.

87 Sarpatwari A, Provan D, Sobnack R, Erqou S, Tai FWD, Sanderson S, Newland A: Autologous ${ }^{111} \mathrm{In}$ labeled platelet sequestration studies in patients with primary immune thrombocytopenia (ITP): a report from the United Kingdom Registry. Blood 2009;114:950, abstr 2406 . 
88 Working Party of the British Committee for Standards in Haematology Clinical Haematology Task Force: Guidelines for the prevention and treatment of infection in patients with an absent or dysfunctional spleen. BMJ 1996;312:430-434.

89 Schiavotto C, Castaman G, Rodeghiero F: Treatment of idiopathic thrombocytopenic purpura (ITP) in patients with refractoriness to or with contraindication for corticosteroids and/or splenectomy with immunosuppressive therapy and danazol. Haematologica 1993;78(suppl 2):29-34.

90 Schoonen WM, Li L, Fryzek JP, Kaye JA: Medical contraindications for splenectomy among adults with immune thrombocytopenic purpura: data from the general parctice research database. Haematologica 2009;94(suppl 2):96, abstr 0244

91 Cooper N, Evangelista ML, Amadori S, Stasi R: Should rituximab be used before or after splenectomy in patients with immune thrombocytopenic purpura. Curr Opin Hematol 2007;14:642-646.

92 Dabak V, Hanbal AI, Kuriakose P: Can rituximab replace splenectomy in immune thrombocytopenic purpura (ITP)? Blood 2007;110(suppl 1, part 1): 393a, abstr 1306

93 Godeau B, Porcher R, Fain O, Lefrère F, Fenaux P, Cheze S, Vekhoff A, Chauveheid MP Stirnemann J, Galicier L, Bourgeois E, Haiat S Varet B, Leporrier M, Papo T, Khellaf M, Michel M, Bierling P: Rituximab efficacy and safety in adult splenectomy candidates with chronic immune thrombocytopenic purpura - results of a prospective multicenter phase 2 study. Blood 2008;112:9991004.

-94 Kuter DJ, Bussel JB, Lyons RM, Pullarkat V, Gernsheimer TB, Senecal FM, Aledort LM, George JN, Kessler CM, Sanz MA, Liebman HA, Slovick FT, de Wolf JT, Bourgeois E, Guthrie TH Jr, Newland A, Wasser JS, Hamburg SI, Grande C, Lefrère F, Lichtin AE, Tarantino MD, Terebelo HR, Viallard JF, Cuevas FJ, Go RS, Henry DH, Redner RL, Rice L, Schipperus MR, Guo DM, Nichol JL: Efficacy of romiplostim in patients with chronic immune thrombocytopenic purpura: a double-blind randomised controlled trial. Lancet 2008;371:395-403.

95 Cheng G, Saleh MN, Bussel JB, Marcher C, Vasey S, Mayer B, Aivado M, Arning M, Stone NL: Oral eltrombopag for the long-term treatment of patients with chronic idiopathic thrombocytopenic purpura: results of a phase III, double-blind, placebo-controlled study (RAISE). Blood 2008;112: 153 , abstr 400

-96 Vesely SK, Perdue JJ, Rizvi MA, Terrell DR, George JN: Management of adult patients with persistent idiopathic thrombocytopenic purpura following splenectomy. Ann Intern Med 2003;140: 112-120.

97 Blanchette M, Freedman J: The history of idiopathic thrombocytopenic purpura (ITP). Transfus Sci 1998;19:231-236

98 Frank E: Die essentielle Thrombopenie. Berl Klin Wochenschr 1915;52:454-458.

99 Heyns AD, Lötter MG, Badenhorst PN, de Kock F, Pieters H, Herbst C, van Reenen OR, Kotze H, Minnaar PC: Kinetics and sites of destruction of 111-indium-oxine-labeled platelets in idiopathic thrombocytopenic purpura: a quantitative study. Am J Hematol 1982;12:167-177.

100 Takahashi R, Sekine N, Nakatake T: Influence of monoclonal antiplatelet glycoprotein antibodies on in vitro human megakaryocyte colony formation and proplatelet formation. Blood 1999;93 1951-1958.
101 Ikeda Y, Miyakawa Y: Development of thrombopoietin receptor agonists for clinical use. J Thromb Haemost 2009;7(suppl 1):239-244.

102 Bussel JB, Cheng G, Saleh MN, Psaila B, Kovaleva L, Meddeb B, Kloczko J, Hassani H, Mayer B, Stone NL, Arning M, Provan D, Jenkins JM: Eltrombopag for the treatment of chronic idiopathic thrombocytpenic purpura. N Engl J Med 2007;357:2237-2247.

103 Bussel JB, Kuter DJ, George JN, McMillan R, Aledort LM, Conklin GT, Lichtin AE, Lyons RM, Nieva J, Wasser JS, Wiznitzer I, Kelly R, Chen CF, Nichol JL: AMG 531, a thrombopoiesis-stimulating protein, for chronic ITP. N Engl J Med 2006;355:1672-1681, Erratum in: N Engl J Med 2006;355:2054.

104 Bussel JB, Kuter DJ, Newland A, de Wolf JTM, Wasser J, Chang P, Nie K, Berger D: Long-term efficacy and safety of romiplostim for the treatment of patients with chronic immune thrombocytopenia (ITP): 5-year update from an openlabel extension study. Blood 2009;114:285, abstr 681

105 Bussel JB, Provan D, Shamsi T, Cheng G, Psaila B, Kovaleva L, Salama A, Jenkins JM, Roychowdhury D, Mayer B, Stone N, Arning M: Effect of eltrombopag on platelet counts and bleeding during treatment of chronic idiopathic thrombocytopenic purpura: a randomised, double-blind, placebo-controlled trial. Lancet 2009;373:641-648.

106 Newland A, Caulier MT, Kappers-Klunne M, Schipperus MR, Lefrere F, Zwaginga JJ, Christal J, Chen CF, Nichol JL: An openlabel, unit dosefinding study of AMG 531, a novel thrombopoiesis-stimulating peptibody, in patients with immune thrombocytopenic purpura. Br J Haematol 2006;135:547-553.

107 Saleh MN, Bussel JB, Cheng G, Meddeb B, Mayer B, Bailey C, Aivado M: Long-term treatment of chronic immune thrombocytopenic purpura with oral eltrombopag: results from the EXTEND study. Blood 2009;114:285, abstr 682.

108 Fogarty PF, Bussel JB, Cheng G, Saleh MN, Meddeb B, Bailey C, Brainsky A: Oral eltrombopag treatment reduces the need for concomitant medications in patients with chronic idiopathic thrombocytopenic purpura. Blood 2008;112:1174, abstr 3424.

109 George JN, Mathias SD, Go RS, Guo M, Henry DH, Lyons R, Redner RL, Rice L, Schipperus MR: Improved quality of life for romiplostimtreated patients with chronic immune thrombocytopenic purpura: results from two randomized, placebo-controlled trials. Br J Haematol 2009; 144:409-415.

110 Grotzinger KM, Matzdorff A, Brown TM, Horblyuk R, Brainsky A: Corticosteroids for the treatment of chronic idiopathic thrombocytopenic purpura: patient-perceived burden. Haematologica 2009;94(suppl 2):96, abstr 0242.

111 Horblyuk R, Matzdorff A, Brown TM, Grotzinger $\mathrm{KM}$, Brainsky $\mathrm{A}$ : Chronic idiopathic thrombocytopenic purpura therapies: the patients' perspective on bothersome effects. Haematologica 2009:94(suppl 2):93, abstr 0236.

112 Matzdorff A, Arnold G: Treatment of chronic immune thrombocytopenic purpura: the patients' perspective. Eur J Haematol 2007;78:381-388.

113 Liebman HA, Henry D, Lefrere F: Long-term safety profile of romiplostim in patients with chronic immune thrombocytopenia (ITP). Blood 2008;112:1171, abstr 3415 .
114 Cheng G, Tarantino M, Gernsheimer T, Meyer O, Brainsky A, Stone NL: Platelet counts following eltrombopag discontinuation in patients with chronic immune thrombocytopenic purpura. Blood 2009;114:1365, abstr 3517.

115 Maddrey WC, Cheng G, Khelif A, Wroblewski SL, Brainsky A: Evaluation of hepatobiliary parameters during eltrombopag treatment in patients with chronic immune thrombocytopenic purpura. Blood 2009;114:951, abstr 2410.

116 Bussel JB, Kuter DJ, Pullarkat V, Lyons RM, Guo M, Nichol JL: Safety and efficacy of longterm treatment with romiplostim in thrombocytopenic patients with chronic ITP. Blood 2009;113: 2161-2171.

117 Tarantino M, Sunkara U, George J, Aledort LM, Guo M, Berger D, Gernsheimer T: Evaluation of bleeding and thrombotic events during long-term use of romiplostim in patients with chronic immune thrombocytopenic purpura. Blood 2008; 112:1177, abstr 3422 .

118 Sarpatwari A, Bennett D, Logie JW, Shukla A, Beach KJ, Newland A, Sanderson S, Provan D: Thromboembolic events among adult patients with idiopathic thrombocytopenic purpura in the United Kingdom General Practice Research Database. Haematologica 2010, in press.

119 Kuter DJ, Bussel JB, Newland A, de Wolf JTM, Guthrie T, Wasser J, Gehl L, Nie K, Berger D Long-term treatment with romiplostim in patients with chronic immune thrombocytopenic purpura (ITP): 3-year update from an open-label extension study. Blood 2008;112:154, abstr 402.

120 Kuter DJ, Mufti G, Brain B, HasserjianR, Rutstein M: Evaluation of bone marrow reticulin formation in romiplostim-treated adult patients with chronic immune thrombocytopenic purpura. Blood 2008;112:1171, abstr 3416

121 Jawa V, Hokom M, Hu Z, El-Abaadi N, Zhuang Y, Berger D, Gupta S, Swanson SJ, Chirmule N: Low immunogenicity of romiplostim in clinical studies with ITP subjects. Ann Hematol 2010, in press.

122 Tomiyama Y, Miyakawa Y, Okamoto S, Katsutani S, Kimura A, Okoshi Y, Ninomiya H, Kosugi H, Nomura S, Ozaki K, Ikeda Y, Koh N, Katsura K, Kanakura Y: Six month treatment of low dose eltrombopag is efficacious in Japanese patients with refractory chronic immune thrombocytopenic purpura (ITP). Blood 2009;114:541, abstr 1324.

123 Arnold DM, Dentali F, Crowther MA, Meyer RM, Cook RJ, Sigouin C, Fraser GA, Lim W, Kelton JG: Systematic review: efficacy and safety of rituximab for adults with idiopathic thrombocytopenic purpura. Ann Intern Med 2007;146:25-33.

124 Medeot M, Zaja F, Vianelli N, Battista M, Baccarani $\mathrm{M}$, Patriarca $\mathrm{F}$, Soldano $\mathrm{F}$, Isola $\mathrm{M}$, De Luca S, Fanin R: Rituximab therapy in adult patients with relapsed or refractory ITP: long term followup results. Eur J Haematol 2008;81:165-169.

125 Parodi E, Rivetti E, Amendola G, Bisogno G, Calabrese R, Farruggia P, Giordano P, Matarese SM, Nardi M, Nobili B, Notarangelo LD, Russo G, Vimercati C, Zecca M, De Mattia D, Ramenghi U: Long-term follow-up analysis after rituximab therapy in children with refractory symptomatic ITP: identification of factors predictive of a sustained response. Br J Haematol 2009; 144:552-558. 
126 Penalver FJ, Jiménez-Yuste V, Almagro M, Alvarez-Larrán A, Rodríguez L, Casado M, Gallur L, Giraldo P, Hernández R, Menor D, Rodríguez MJ, Caballero D, González R, Mayans J, Millán I, Cabrera JF, on behalf of the Multi-Institutiona Retrospective Spanish Study on the use of rituximab in refractory ITP: Rituximab in the management of chronic immune thrombocytopenic purpura: an effective and safe therapeutic alternative in refractory patients. Ann Hematol 2006; 85:400-406.

127 El-Najjar I, Rule SAJ, Nokes TCJ: A fixed, low dose of anti-CD20 antibody (rituximab) is an effective treatment for patients with chronic immune thrombocytopenia. Br J Haematol 2006; 133(suppl 1):79, abstr 196.

128 Provan D, Butler T, Evangelista ML, Amadori S, Newland AC, Stasi R: Activity and safety profile of low-dose rituximab for the treatment of autoimmune cytopenias in adults. Haematologica 2007;92:1695-1698.

-129 Provan D, Newland AC, Amadori S, Stasi R: Rituximab in the treatment of autoimmune haematological disorders. Br J Haematol 2008;143: 294.

130 Carson KR, Evens AM, Richey EA, Habermann TM, Focosi D, Seymour JF, Laubach J, Bawn SD, Gordon LI, Winter JN, Furman RR, Vose JM, Zelenetz AD, Mamtani R, Raisch DW, Dorshimer GW, Rosen ST, Muro K, Gottardi-Littell NR, Talley RL, Sartor O, Green D, Major EO, Bennett CL: Progressive multifocal leukoencephalopathy after rituximab therapy in HIV negative patients: a report of 57 cases from the Research on Adverse Drug Events and Reports project. Blood 2009;113:4834-4840.

131 Fleischmann RM: Progressive multifocal leukoencephalopathy following rituximab treatment in a patient with rheumatoid arthritis. Arthritis Rheum 2009;60:3225-3228.

$\checkmark 132$ Bouroncle BA, Doan CA: Refractory idiopathic thrombocytopenic purpura treated with azathioprine. N Engl J Med 1966;275:630-635.

133 Sussman LN: Azathioprine in refractory idiopathic thrombocytopenic purpura. JAMA 1967 : 202:259-263.

134 Emilia G, Morselli M, Luppi M, Longo G, Marasca R, Gandini G, Ferrara L, D'Apollo N, Potenza L, Bertesi M, Torelli G: Long-term salvage therapy with cyclosporin A in refractory idiopathic thrombocytopenic purpura. Blood 2002;99:1482-1485.

135 Meran JG, Freund M, Kessen-Albers M, Ganser A, Ludwig H: Cyclosporin A (CsA) in steroidrefractory, chronic idiopathic immune-thrombocytopenia. Onkologie 1999;22(suppl 1):121, abstr 0452 .

136 Maloisel F, Andres E, Zimmer J, Noel E, Zamfir A, Koumarianou A, Dufour P: Danazol therapy in patients with chronic idiopathic thrombocytopenic purpura: long-term results. Am J Med 2004; 116:590-594.

137 Godeau B, Durand JM, Roudot-Thoraval F, Tennezé A, Oksenhendler E, Kaplanski G, Schaeffer A, Bierling P: Dapsone for chronic autoimmune thrombocytopenic purpura: a report of 66 cases. Br J Haematol 1997;97:336-339.

-138 Hernandez F, Linares M, Colomina P, Pastor E, Cerveró A, Pérez A, Perella M: Dapsone for refractory chronic idiopathic thrombocytopenic purpura. Br J Haematol 1995;90:473-475.
139 Vancine-Califani SM, De Paula EV, Ozelo MC, Orsi FL, Fabri DR, Annichino-Bizzacchi JM: Efficacy and safety of dapsone as a second-line treatment in non-splenectomized adults with immune thrombocytopenic purpura. Platelets 2008;19:489-495.

140 Fibich C, Fiedler F, Herold M, Kegel T, Schöber C, Schmoll HJ: Mycophenolate-mofetil (Cellcept $\left.^{\circledR}, \mathrm{MMF}\right)$ in the treatment of steroid-refractory autoimmune thrombocytopenia and Evanssyndrome. Blood 1998;92(suppl 1, part 1):177a, abstr 716 .

141 Howard J, Hoffbrand AV, Prentice HG, Mehta A: Mycophenolate mofetil for the treatment of refractory auto-immune haemolytic anaemia and auto-immune thrombocytoepnia purpura. $\mathrm{Br} \mathrm{J}$ Haematol 2002;117:712-715.

142 Provan D, Moss AJ, Newland AC, Bussel JB: Efficacy of mycophenolate mofetil as single-agent therapy for refractory immune thrombocytopenic purpura. Am J Hematol 2006;81:19-25.

143 Linares M, Cervero A, Sanchez M, Garcia S, Miguel-Sosa A, Miguel-Garcia A, Miguel-Borja JM: Slow infusion of vincristine in the treatment of refractory thrombocytopenic purpura. Acta Haematol 1988;80:173-174.

144 Manoharan A: Slow infusion of vincristine in the treatment of idiopathic thrombocytopenic purpura. Am J Hematol 1986;21:135-138.

145 Marmont AM, Damasio EE, Gori E: Vinblastin sulphate in idiopathic thrombocytopenic purpura. Lancet 1971;2:94.

146 Zeile G: Vincristin-therapy of chronic refractory idiopathic thrombocytopenic purpura. Blut 1978; 36:217-223.

147 Lim SH, Hale G, Marcus RE, Waldmann H, Baglin TP: CAMPATH-1 monoclonal antibody therapy in severe refractory autoimmune thrombocytopenic purpura. Br J Haematol 1993;84:542-544.

148 Willis F, Marsh JC, Bevan DH, Killick SB, Lucas G, Griffiths R, Ouwehand W, Hale G, Waldmann H, Gordon-Smith EC: The effect of treatment with Campath-1H in patients with autoimmune cytopenias. Br J Haematol 2001;114:891-898.

149 Boruchov DM, Gururangan S, Driscoll MC, Bussel JB: Multiagent induction and maintenance therapy for patients with refractory immune thrombocytopenic purpura (ITP). Blood 2007; 110:3526-3531.

150 Figueroa M, Gehlsen J, Hammond D, Ondreyco S, Piro L, Pomeroy T, Williams F, McMillan R: Combination chemotherapy in refractory immune thrombocytopenic purpura. N Engl J Med 1993;328:1226-1229.

151 Gururangan S, Bussel JB: Combination immunotherapy for patients with refractory ITP or Evan's syndrome (ES). Blood 1998;92(suppl 1, part 2): $84 b$, abstr 3345.

152 Arnold DM, Nazi I, Santos A, Chan H, Heddle NM, Warkentin TE, Kelton JG: Combination immunosuppressant therapy for patients with chronic refractory immune thrombocytopenic purpura. Blood 2010;115:29-31.

153 Huhn RD, Fogarty PF, Nakamura R, Read EJ, Leitman SF, Rick ME, Kimball J, Greene A, Hansmann K, Gratwohl A, Young N, Barrett AJ, Dunbar CE: High-dose cyclophosphamide with autologous lymphocyte-depleted peripheral blood stem cell (PBSC) support for treatment of refractory chronic autoimmune thrombocytopenia. Blood 2003;101:71-77.
154 Hurtado RM, Myrna CH, Silmia CM, Juan SM, Juan OC, Manuel BR, Carolina GG, Pablo VV, Miguel PJ, Arturo L: Autologous stem cell transplantation for autoimmune disorders, a case report. Blood 1999;94(suppl 1, part 2):401b, abstr 5019.

155 Miranda M, Reich L, Traynor A, Young J, Papadopoulas E, Fishman M, Welles C, Burt RK: Cure of Evans syndrome (autoimmune hemolytic anemia and idiopathic thrombotic thrombocytopenia) by allogeneic hematopoietic stem cell transplantation. Blood 1999;94(suppl 1, part 2): $384 b$, abstr 4948.

156 Nakamura R, Huhn RD, Read EJ, Rick M, Leitman SF, Greene A, Gratwohl A, Young NS, Barrett AJ, Dunbar CE: Intensive immunosuppression with high-dose cyclophosphamide and autologous CD34+ selected hematopoietic cell support for chronic refractory autoimmune thrombocytopenia (AITP): interim report. Blood 1999;94(suppl 1, part 1):646a, abstr 2869.

157 Mannucci PM: Hemostatic drugs. N Engl J Med 1998;339:245-253.

158 British Committee for Standards in Haematology, Blood Transfusion Task Force: Guidelines for the use of platelet transfusions. Br J Haematol 2003; 122:10-23.

159 Bundesärztekammer: Querschnitts-Leitlinien (BÄK) zur Therapie mit Blutkomponenten und Plasmaderivaten. 4. überarbeitete Auflage. Köln, Deutscher Ärzteverlag, 2009. Leitlinien abrufbar unter www.baek.de/haemotherapie.

160 Greinacher A, Kiefel V, Klüter H, Kroll H, Pötzsch B, Riess H: Empfehlungen zur Thrombozytentransfusion der Thrombozyten-Arbeitsgruppe der DGTI, GTH und DGHO. Transfus Med Hemother 2006;33:528-543.

161 Samama CM, Djoudi R, Lecompte T, NathanDenizot N, Schved JF; AFSSAPS Expert Group: Perioperative platelet transfusion: recommendations of the Agence Francaise de Securite Sanitaire des Produits de Sante (AFSSaPS) 2003. Can J Anaesth 2005;52:30-37.

162 Nakayama T, Onoda K: Vaccine adverse events reported in post-marketing study of the Kitasato Institute from 1994 to 2004. Vaccine 2007;25:570 576.

163 Shu M, Liu Q, Wang J, Ao R, Yang C, Fang G, Wan C, Guo W: Measles vaccine adverse events reported in the mass vaccination campaign of Sichuan province, China from 2007 to 2008 . Vaccine 2009 , in press.

164 Rajantie J, Zeller B, Treutiger I, Rosthöj S; NOPHO ITP Working Group and five national study groups: Vaccination associated thrombocytopenic purpura in children. Vaccine 2007;25: 1838-1340.

165 Miller AB, Hoogstraten B, Staquet M, Winkler A: Reporting results of cancer treatment. Cancer 1981;47:207-214.

166 Heddle NM, Cook RJ, Tinmouth A, Tinmouth A, Kouroukis T, Hervig T, Klapper E, Brandwein JM, Szczepiorkowski ZM, AuBuchon JP, Barty RL; Lee KA for the SToP Study investigators of the BEST Collaborative: A randomized controlled trial comparing standard and lowdose strategies for transfusion of platelets (SToP) to patients with thrombocytopenia. Blood 2009;113:1564-1573.

167 National Cancer Institute: NCI Common Terminology Criteria for Adverse Events: CTCAE, version MedDRA v9.0. www.cancer.gov (überprüft Jan 2010). 